\title{
Implantación de las Unidades SI en los resultados de laboratorio clínico Especificaciones de estilo y tablas de conversión
}

\author{
D.S. Young
}

Las unidades SI se usan actualmente en muchos países para informar los resultados de laboratorio clínico. Entre 1987 y 1988 se adoptarán estas unidades para reemplazar las que se han empleado hasta el momento con ese fin en los Estados Unidos. Esta acción, que ha sido promovida por el Grupo Médico y Coordinador de Salud del Consejo Métrico Nacional Americano y avalada por muchas sociedades profesionales que incluyen a la Asociación Médica Americana, conducirá a que los informes de laboratorio se hagan en términos molares utilizando el litro como la referencia de volumen.

\section{¿Qué son las Unidades SI?}

“Unidades SI" es la abreviatura "Le Système Internacional d'Unités". Estas unidades son el resultado de mas de un siglo de cooperación internacional para desarrollar un sistema de unidades de medida que sea aceptable universalmente. La unidad SI es una consecuencia del sistema métrico que ha sido ampliamente utilizado en la mayor parte del mundo.

Sistema Internacional de unidades, nombre adoptado por la XI Conferencia General de Pesas y Medidas (celebrada en París en 1960) para un sistema universal, unificado y coherente de unidades de medida, basado en el sistema mks (metro-kilogramo-segundo). Este sistema se conoce como SI, iniciales de Sistema Internacional. En la Conferencia de 1960 se definen patrones para seis unidades básicas y dos unidades suplementarias (radián y estereorradián). En 1971 se agrega una séptima unidad, el mol. Las dos unidades suplementarias se suprimen como una clase independiente dentro del Sistema Internacional en la XX Conferencia General de Pesas y Medidas (1995); estas dos unidades quedaron incorporadas al SI como unidades derivadas sin dimensiones.

La Oficina Internacional de Pesas y Medidas (BIPM) fue creada por la Convención del Metro, firmada en París el 20 de mayo de 1875 por diecisiete Estados, en la última sesión de la Conferencia Diplomática. Esta Convención fue modificada en 1921.

El BIPM tiene su sede cerca de París, en los terrenos $\left(43520 \mathrm{~m}^{2}\right)$ del Pabellón de Breteuil (Parque de Saint-Cloud), puesto a su disposición por el Gobierno Francés; su mantenimiento se financia conjuntamente por los Estados Miembros de la Convención del Metro.

La misión del BIPM es asegurar la unificación mundial de las medidas; por lo tanto se encarga de:

- establecer los patrones fundamentales y las escalas para la medida de las principales magnitudes físicas y conservar los prototipos internacionales;

- llevar a cabo comparaciones de los patrones nacionales e internacionales;

- asegurar la coordinación de las técnicas de medida correspondientes;

- efectuar y coordinar las mediciones de las constantes físicas fundamentales relevantes en las actividades precedentes.

La referencia es asi: Oficina Internacional de pesas y Medidas. Sistema Internacional de Unidades SI. 2da ed. en español. Centro Español de metrología; 2008.

Finalmente, el SI cuenta con siete unidades básicas de las que se derivan otras unidades. Estos siete pilares a partir de los cuales se han desarrollado las otras unidades se listan en la Tabla 1, junto con la propiedad o cantidad física a la que hacen referencia y a su símbolo oficial. Es posible combinar estas bases con el fin de expresar alguna otra propiedad, pero, por simplicidad, a las unidades derivadas se les da un nombre especial. En la Tabla 2 aparecen algunos ejemplos representativos.

Si para informar los datos de laboratorio se emplearán únicamente las unidades básicas

Traducción de Young DS. "Implantation of SI Units for Clinical Laboratory Data-Style specifications and conversión Tables" Ann Intern Med 1987; 106: 114-129.

Este documento no tiene derechos de autor y su reproducción es libre y fue autorizado, por Faiver Ramirez.

Acta Med Colomb 1987; 12:395-410. 
Tabla 1. Unidades básicas de SI.

\begin{tabular}{|lcc|}
\hline Cantidad física & Unidad de base & Símbolo SI \\
\hline Longitud & Metro & $\mathrm{m}$ \\
Masa & kilogramo & $\mathrm{kg}$ \\
Tiempo & segundo & $\mathrm{s}$ \\
Cantidad de sustancia & Mol & $\mathrm{mol}$ \\
Temperatura & & $\mathrm{K}$ \\
Termodinámica & Kelvin & $\mathrm{A}$ \\
Corriente eléctrica & amperio & $\mathrm{cd}$ \\
Intensidad luminosa & candela & \\
\hline
\end{tabular}

algunos valores serían extremadamente grandes o pequeños. El SI utiliza una serie de prefijos antes de la unidad básica para formar múltiplos y submúltiplos decimales. Los múltiplos y submúltiplos preferidos cambia la cantidad por incrementos de $10^{3} \mathrm{o} 10^{-3}$ como se ilustra en la Tabla 3 , pero ocasionalmente se emplean algunos incrementos o decrementos menores que estos factores. Estas excepciones se incluyen en la zona encuadrada de la tabla.

El símbolo del prefijo debe escribirse como aparece en la tabla unido a la unidad sin ningún espacio entre ellos: así, kilopascal se escribe $\mathrm{kPa}$. Por razones históricas la unidad básica de masa es el kilogramo $(\mathrm{kg})$. No se debe combinar ningún prefijo con esta unidad básica. Así, 1.000 kilogramos se expresa como un megagramo $(\mathrm{Mg})$ en lugar de 1 kilokilogramo. Aunque el símbolo correcto de micro es la $\mu$, se propone que se acepte la "u" corriente para utilizar los sistemas automáticos de procesamiento de datos actualmente en uso.

El SI conlleva ciertas normas de estilo. Así, los símbolos se expresan en letras minúsculas excepto cuando el nombre de una unidad se deriva de un nombre propio. Cuando se escribe en forma completa una unidad derivada de un nombre propio, no se usa mayúscula ni aún en la primera letra. La unidad de presión es el pascal, pero se le abrevia Pa. Sin embargo, se acepta "L" como símbolo del litro aunque no es un nombre propio, Esta variación de la práctica normal se hace para evitar la confusión entre la letra minúscula "l" y el numeral "1" que tienen símbolos muy similares en algunos tipos de caracteres. Los símbolos SI nunca se acompañan de punto, excepto al final de una frase, y nunca se expresan en plural. Un nombre nunca debe combinarse con un símbolo. Cuando se utilizan juntos dos o más nombres o símbolos, éstos deben deletrearse en forma completa o en forma abreviada, pero cuando se imprimen valores medidos se prefiere usar los símbolos de las unidades en lugar de sus nombres completos. En este caso se debe dejar un espacio entre el numeral y el símbolo -por ejemplo, $50 \mathrm{~mL}$ y $37^{\circ} \mathrm{C}$. El producto de dos o más unidades se indica con un punto encima de la línea para distinguirlo del punto decimal que se coloca en la línea. Se debe colocar un cero antes del punto decimal en un valor numérico y se deben preferir los números decimales en lugar de las fracciones. Deben emplearse espacios para separar números largos en segmentos de 3 en ambas direcciones a partir del punto
Tabla 2. Unidades derivadas representativas.

\begin{tabular}{|llc|}
\hline Unidad derivada & Nombre y Símbolo & $\begin{array}{c}\text { Derivación de las } \\
\text { unidades básicas }\end{array}$ \\
\hline Área & metro cuadrado & $\mathrm{m}^{2}$ \\
Volumen & metro cúbico & $\mathrm{m}^{3}$ \\
Fuerza & newton $(\mathrm{N})$ & $\mathrm{kg} \cdot \mathrm{m} \cdot \mathrm{s}^{-2}$ \\
Presión & pascal $(\mathrm{Pa})$ & $\mathrm{kg} \cdot \mathrm{m}^{-2} \cdot \mathrm{s}^{-2}\left(\mathrm{~N} / \mathrm{m}^{2}\right)$ \\
trabajo, energía & julio $(\mathrm{J})$ & $\mathrm{kg} \cdot \mathrm{m}^{2} \cdot \mathrm{s}^{-2}(\mathrm{~N} \cdot \mathrm{m} \cdot)$ \\
densidad de masa & kilogramo por metro cúbico & $\mathrm{kg} \cdot / \mathrm{m}^{3}$ \\
Frecuencia & hertz $(\mathrm{Hz})$ & $\mathrm{s}$ \\
\hline
\end{tabular}

Tabla 3. prefijos y símbolos de los múltiplos y submúltiplos decimales*.

\begin{tabular}{|lcc|}
\hline Factor & Prefijo & Símbolo \\
\hline $10^{18}$ & exa & $\mathrm{E}$ \\
$10^{15}$ & peta & $\mathrm{P}$ \\
$10^{12}$ & tera & $\mathrm{T}$ \\
$10^{9}$ & giga & $\mathrm{G}$ \\
$10^{6}$ & mega & $\mathrm{M}$ \\
$10^{3}$ & kilo & $\mathrm{K}$ \\
& & \\
$10^{2}$ & hecto & $\mathrm{H}$ \\
$10^{1}$ & deca & $\mathrm{Da}$ \\
$10^{-1}$ & deci & $\mathrm{D}$ \\
$10^{-2}$ & centi & $\mathrm{C}$ \\
& & \\
$10^{-3}$ & mili & $\mathrm{M}$ \\
$10^{-6}$ & micro & $\mu$ \\
$10^{-9}$ & nano & $\mathrm{N}$ \\
$10^{-12}$ & pico & $\mathrm{P}$ \\
$10^{-15}$ & femto & $\mathrm{F}$ \\
$10^{-18}$ & atto & $\mathrm{A}$ \\
* Los factores que se incluyen en el rectángulo no llenan los requisitos de los cambios \\
incrementados de $10^{3}$ y de $10^{-3}$, pero aún se emplean fuera del campo médico. \\
\hline
\end{tabular}

decimal; es opcional el empleo de un espacio en los números de cuatro dígitos. No se deben emplear comas para evitar posibles confusiones con el punto decimal que todavía se indica por una coma en algunos países europeos. Estos requisitos de estilo se presentan resumidos en la Tabla 4.

\section{Unidades SI en medicina}

La razón lógica para informar los resultados de las pruebas de laboratorio en Unidades SI es que se trata de una práctica común en muchos países del mundo donde se emplean rutinariamente en los asuntos de atención médica y en las publicaciones de investigación y otros estudios. Sin embargo, la razón dominante del cambio es que los componentes biológicos reaccionan in vivo con base molar. Así, será posible una mejor comprensión de las cantidades relativas de constituyentes de los fluidos corporales y de los procesos biológicos y sus interrelaciones entre los diferentes ciclos metabólicos si todos los resultados se expresan en unidades uniformes relativas a la cantidad real de reactivos en moles.

Cuando el resultado se expresa en unidades de concentración de masa, por ejemplo mg, sin tener en cuenta el 
Tabla 4. Especificaciones sobre estilo de la unidades SI.

\begin{tabular}{|c|c|c|c|}
\hline Especificaciones & Ejemplo & Estilo Incorrecto & Estilo Correcto \\
\hline Use letras minúsculas para los símbolos o abreviaturas & kilogramo & $\mathrm{Kg}$ & $\mathrm{kg}$ \\
\hline \multirow[t]{3}{*}{ Excepciones } & kelvin & $\mathrm{k}$ & $\mathrm{K}$ \\
\hline & amperio & a & A \\
\hline & litro & 1 & $\mathrm{~L}$ \\
\hline \multirow[t]{2}{*}{ No se deben poner punto después del símbolo } & metro & m. & $\mathrm{m}$ \\
\hline & mole & mol. & mol \\
\hline \multicolumn{4}{|l|}{ Excepción: al final de cada frase } \\
\hline Los símbolos no se representan en plural & kilogramos & kgs & $\mathrm{kg}$ \\
\hline & metros & $\mathrm{ms}$ & $\mathrm{m}$ \\
\hline No debe combinarse nombres y símbolos & fuerza & kilogramo.metro. $\mathrm{s}^{-2}$ & $\mathrm{~kg} \cdot \mathrm{m} \cdot \mathrm{s}^{-2}$ \\
\hline \multirow[t]{2}{*}{ Cuando se utilizan números se deben preferir los símbolos } & & 100 metros & $100 \mathrm{~m}$ \\
\hline & & 2 moles & $2 \mathrm{~mol}$ \\
\hline Debe dejarse un espacio entre el número y el símbolo & & $50 \mathrm{ml}$ & $50 \mathrm{~mL}$ \\
\hline El producto de las unidades se indica mediante un punto encima de la línea & & $\mathrm{kg} \mathrm{x} \mathrm{m/ \textrm {s } ^ { 2 }}$ & kg.m.s $-^{2}$ \\
\hline Se debe emplear únicamente una diagonal (/) por expresión & & $\mathrm{mmol} / \mathrm{L} / \mathrm{s}$ & $\mathrm{mmo} /(\mathrm{L} . \mathrm{s})$ \\
\hline Utilice siempre cero antes del decimal & & .01 & 0.01 \\
\hline Deben emplearse decimales en lugar de fracciones & & $3 / 4,75 \%$ & 0.75 \\
\hline Deben emplearse espacios para separar los números largos & & $1,500,000$ & 1500000 \\
\hline Excepción: es opcional con los números de cuatro dígitos & & 1,000 & 1000 ó 1000 \\
\hline
\end{tabular}

denominador o la unidad de concentración, las cantidades relativas de la sustancia analizada no son claras; habría aparentemente 200 veces más albúmina que bilirrubina en un neonato ictérico que tiene una concentración sérica de bilirrubina de $20.0 \mathrm{mg} / \mathrm{dL}$ y una concentración de albúmina de $4.0 \mathrm{~g} / \mathrm{dL}$. Sin embargo únicamente hay 1.7 veces más de la misma concentración si se expresa en términos molares, $340 \mathrm{umol} / \mathrm{L}$ de bilirrubina y $580 \mathrm{umol} / \mathrm{L}$ para la albúmina. La influencia de la concentración de albúmina en la unión de la bilirrubina y otros compuestos se hace mucho más aparente, así como el desplazamiento de un compuesto por el otro.

Con el empleo del SI es posible relacionar las cantidades de reactivos en los diferentes ciclos metabólicos que comprenden, por ejemplo, los aminoácidos, los carbohidratos y las grasas. También es posible ver la asociación de compuestos o metabolitos que se relacionan entre sí en diferentes tejidos, por ejemplo la hemoglobina en los eritrocitos y el plasma, la bilirrubina y el hierro en el plasma, en conjunto con el urobilinógeno en la orina y las heces.

\section{Influencia de las Unidades SI en la práctica médica corriente}

Aunque el SI permite informar los datos de laboratorio en términos de concentración de masa, el uso de unidades de masa tales como mg/L no indica las cantidades fisiológicamente importantes del producto analizado. Para lograr esto se deben emplear unidades relacionadas con la cantidad de una sustancia. Estas se expresan en términos de moles por litro. Aunque por derivación la unidad de referencia, el volumen debería ser el metro cúbico $\left(\mathrm{m}^{3}\right)$, se ha aceptado en la actualidad como referencia el litro
(L) como nombre especial para el decímetro cúbico $\left(\mathrm{dm}^{3}\right)$. La principal modificación en el informe de los resultado de laboratorio es el referir todos los valores al litro en lugar de unidades tales como el microlito o el decilitro, y el concepto de cantidad de sustancia que implica que un compuesto medido será descrito en moles o sus unidades en lugar de términos de masa, por ejemplo gramos o miligramos. Cuando se desconoce la masa molecular de un compuesto, su concentración podrá informarse en términos de masa (ver en la Tabla 2 del apéndice). El SI emplea el pascal $(\mathrm{Pa})$ como la unidad de presión, pero no se recomienda su uso para informar la presión sanguínea en el momento actual; sin embargo, se le acepta como unidad para expresar las presiones parciales de gases en vez de milímetros de $\mathrm{Hg}$. El segundo (s) es la unidad de referencia del tiempo ya que es una unidad básica SI y se emplea en lugar del minuto. Aunque no es una unidad SI el día (d) aún se acepta para designar largos períodos de tiempo en lugar de los múltiplos del segundo. Se ha propuesto la unidad katal, abreviado como kat y que corresponde a $\mathrm{mol} / \mathrm{s}$, como un nombre especial para designar la actividad catalítica. El Grupo Médico y Coordinador de Salud del Consejo Métrico Nacional Americano recomienda el uso de kat/L para informar la actividad enzimática, debido a la complejidad de las unidades oficiales que son difíciles de escribir y de imprimir en el computador. La nueva unidad kat/L reemplazará las unidades por litro (U/L) así como las múltiples unidades basadas en nombres individuales. Para aquellas sustancias cuya actividad se informa en términos de unidades biológicas estandarizadas -por ejemplo hormona- continuarán usándose las mismas unidades, pero con el litro como referencia de volumen. 
Así, el impacto inmediato de la introducción de estas nuevas unidades en los informes de laboratorio en la práctica médica corriente, estará dado por la presentación de números que no serán inicialmente familiares al médico.

Cuando se empleen unidades de concentración de masa, los valores serán generalmente diez veces mayores que los que el médico está acostumbrado a manejar. La implantación de las unidades SI en muchos otros países no se ha asociado con problema para los pacientes. Tampoco lo constituirá entre nosotros si se tiene en cuenta que todos los valores numéricos se asocien inequívocamente con sus unidades. Adicionalmente se recomienda que los valores numéricos en las muestras de los pacientes se presenten con el rango o intervalo apropiado de referencia (valores normales), por lo menos hasta que los médicos se hayan acostumbrado al nuevo sistema SI.

\section{Hematología}

\section{Situaciones especiales}

Con el litro como la medida actualizada de referencia volumétrica, los valores de recuento celular aumentarán $10^{6} \mathrm{del}$ obtenido cuando el $\mathrm{mm}^{3}$ era el volumen de referencia. Sin embargo, los valores numéricos serán los mismos si se incluye el factor de multiplicación como parte de la unidad -por ejemplo, X 10\%/L. En relación con un informe sobre concentraciones de hemoglobina, sobre lo cual ha habido mucha discusión debido a que se apoya tanto la expresión del monómero, $\mathrm{Hb}$ (Fe) como el tetrámero, $\mathrm{Hb}(4 \mathrm{Fe})$, el Comité Internacional para la estandarización en Hematología favorece la expresión de la hemoglobina como la concentración de la sustancia en términos del monómero. Por lo menos al presente, el Grupo Medico y el Coordinador de Salud recomiendan el uso continuado de medidas de masa de concentración de hemoglobina, de manera que la unidad de informe sería el g/L.

Con el SI, el concepto de fracción numérica reemplaza el porcentaje. Así, se emplea la unidad 1 para reemplazar las antiguas unidades representativas de fracción de masa, fracción de volumen o cantidades relativas. El hematocrito de $45 \%$ se informaría como 0.45 . Cuando se efectúa electroforesis de hemoglobina y otros constituyentes de la sangre, se debe reportar cada componente en términos de fracción de masa - por ejemplo, 0.20 en lugar de $20 \%$. Igualmente el recuento diferencial de células en un extendido periférico o de médula ósea, debe expresarse como una fracción numérica del total de células contadas.

\section{Microbiología}

Las medidas de antibióticos tanto en el suero como en concentraciones inhibitorias del crecimiento de organismos en el laboratorio clínico, se informarán en unidades molares tal como se hace con otras drogas determinadas en el laboratorio. El recuento de colonias se debe referir al litro como unidad de volumen; el valor numérico permanecerá sin cambios. El recuento de bacterias en medios sólidos se debe referir al gramo como unidad denominador.

\section{Química clínica}

Aunque se continúen empleando las unidades de masa para la administración de medicamentos, su concentración en los fluidos corporales se debe informar en términos molares, de manera que se pueda entender en relación con la concentración de otras sustancias analizadas. A la larga se anticipa que los medicamentos también deberán ser administrados en cantidades molares. Es muy posible que la diferencia entre las unidades de administración y de medición de las drogas no conduzca a confusión ya que la cantidad de droga absorbida y activa in vivo es sustancialmente diferente de la que se ingirió si se administró por vía oral. Más razón se encuentra para emplear las unidades molares cuando las drogas se administran por vía intramuscular o intravenosa.

Aunque no es una unidad SI, el osmol debe continuar empleándose para las medicas de presión osmótica; los resultados se deben expresar en términos de mol/ $\mathrm{kg}$. La concentración de hormonas debe informarse en términos de concentración de sustancia cuando sea posible, pero si se informa en términos de actividad biológica se deben usar las unidades internacionales empleadas anteriormente, pero con el litro como referencia de volumen. Cuando se conoce la masa molecular de las hormonas o proteínas las concentraciones de estas sustancias deben informarse en moles, en lugar de gramos o submúltiplos.

\section{Factores de conversión}

En las Tablas 1 y 2 del apéndice aparecen los nombres de las pruebas y los fluídos en que se hicieron sus determinaciones, junto con los valores típicos de rango o intervalos de referencia en las unidades tradicionales. Se presenta el factor de multiplicación para convertir las unidades métricas corrientes en unidades SI, lo mismo que el intervalo de referencia en unidades SI con el símbolo correcto. Para convertir las unidades SI en las unidades convencionales el valor numérico de aquellas se divide por el factor de conversión. En las tablas del índice también aparece el número apropiado de dígitos significativos y se sugieren los incrementos mínimos para informar, de manera que no implique una mayor precisión de la que actualmente se logra mediante las unidades SI. Los intervalos y los incrementos sugeridos se deben al nivel de imprecisión presente en el procedimiento analítico pertinente y no son necesariamente de relevancia clínica.

Deben emplearse los nombres enunciados de cada prueba de laboratorio en lugar de los que se usan ocasionalmente, ya que ellos reflejan el estado de la sustancia analizada en condiciones fisiológicas - por ejemplo, debe decirse lactato de hidrogenasa en lugar de hidrogenasa láctica, y piruvato en vez de ácido pirúvico.

Es preciso notar que los intervalos de referencia que se citan en las tablas del apéndice, particularmente aquellos relacionados con determinaciones enzimáticas, pueden depender del método que se practique y no deben aplicarse indiscriminadamente a los valores obtenidos en cualquier laboratorio sin las verificaciones correspondientes. 


\section{APENDICE}

Tabla 1. Tabla de conversión SI para valores en hematología clínica

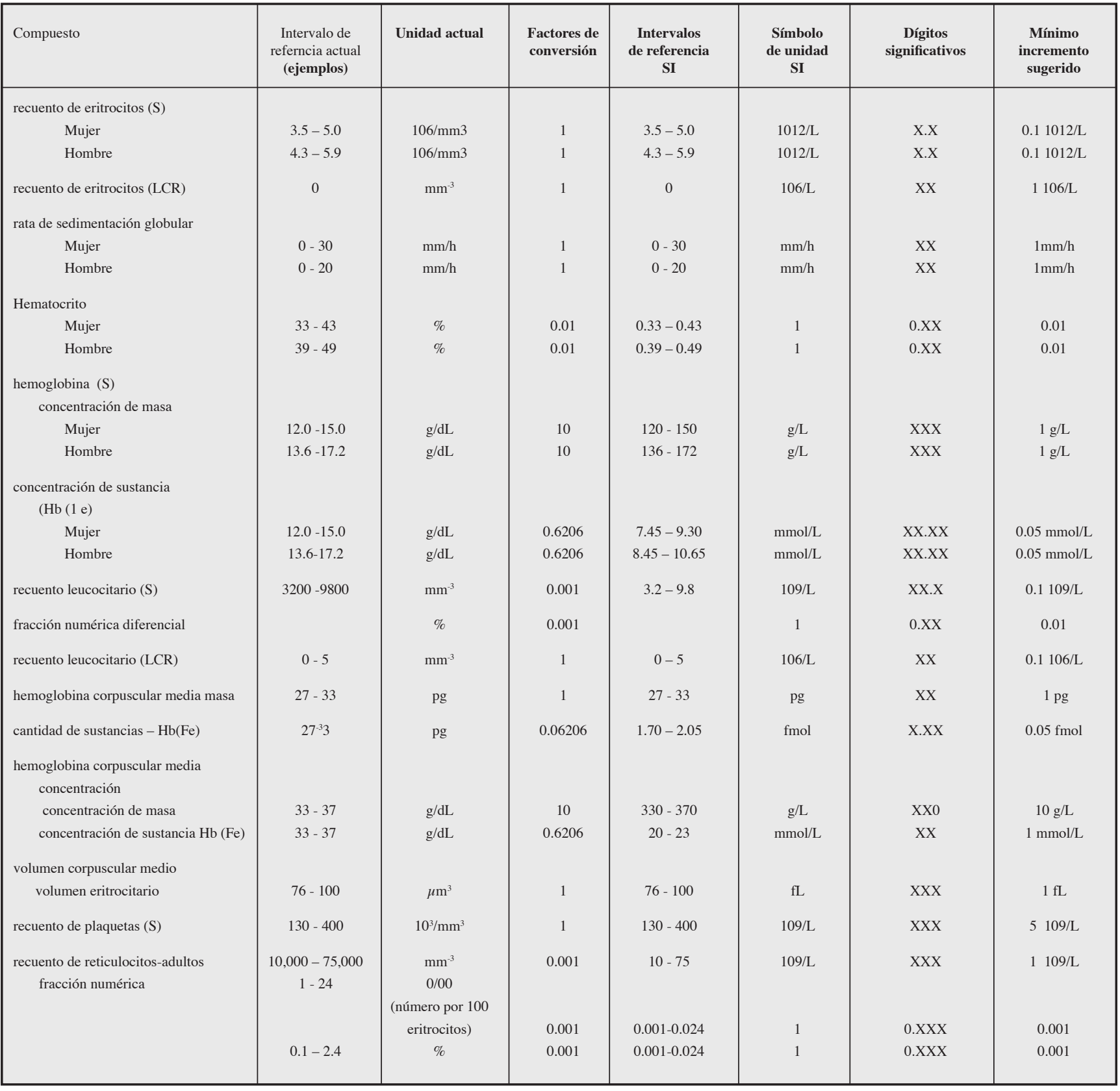


APENDICE

Tabla 2. Factores de conversión para los resultados de química clínica.

\begin{tabular}{|c|c|c|c|c|c|c|c|}
\hline Compuesto & $\begin{array}{c}\text { Intervalo de } \\
\text { referencia actual }\end{array}$ & $\begin{array}{l}\text { Unidad } \\
\text { actual }\end{array}$ & $\begin{array}{l}\text { Factores de } \\
\text { conversión }\end{array}$ & $\begin{array}{c}\text { Intervalos } \\
\text { de referencia } \\
\text { SI }\end{array}$ & $\begin{array}{l}\text { Símbolo de } \\
\text { unidad } \\
\text { SI }\end{array}$ & $\begin{array}{l}\text { Dígitos } \\
\text { significativos }\end{array}$ & $\begin{array}{c}\text { Mínimo } \\
\text { incremento } \\
\text { sugerido }\end{array}$ \\
\hline acetaminofen $(\mathrm{P})$ - tóxico & $>5.0$ & $\mathrm{mg} / \mathrm{dL}$ & 66.16 & $>330$ & $\mu \mathrm{mol} / \mathrm{L}$ & XX0 & $10 \mu \mathrm{mol} / \mathrm{L}$ \\
\hline acetoacetato $(\mathrm{S})$ & $0.3-3.0$ & $\mathrm{mg} / \mathrm{dL}$ & 97.95 & $30-300$ & $\mu \mathrm{mol} / \mathrm{L}$ & $\mathrm{XX} 0$ & $10 \mu \mathrm{mol} / \mathrm{L}$ \\
\hline acetona $(\mathrm{S}, \mathrm{s})$ & 0 & $\mathrm{mg} / \mathrm{dL}$ & 172.2 & 0 & $\mu \mathrm{mol} / \mathrm{L}$ & XX0 & $10 \mu \mathrm{mol} / \mathrm{L}$ \\
\hline fosfatasa ácida (s) & $0-5.5$ & $\mathrm{U} / \mathrm{L}$ & 16.67 & $0-90$ & Nkat/L & $\mathrm{XX}$ & 2 nkat/L \\
\hline adrenocorticotrofina (ACTH) (P) & $20-100$ & $\mathrm{Pg} / \mathrm{mL}$ & 0.2202 & $4-22$ & $\mathrm{pmol} / \mathrm{L}$ & $\mathrm{XX}$ & $1 \mathrm{pmol} / \mathrm{L}$ \\
\hline alanina aminotransferasa (s) & $0-35$ & $\mathrm{U} / \mathrm{L}$ & 0.01667 & $0-0.58$ & $\mu \mathrm{kat} / \mathrm{L}$ & X.XX & $0.02 \mu \mathrm{kat} / \mathrm{L}$ \\
\hline albúmina (s) & $4.0-6.0$ & $\mathrm{~g} / \mathrm{dL}$ & 10.0 & $40-60$ & $\mathrm{~g} / \mathrm{L}$ & XX & $1 \mathrm{~g} / \mathrm{L}$ \\
\hline aldolasa (s) & $0-6$ & $\mathrm{U} / \mathrm{L}$ & 16.67 & $0-100$ & nkat/L & XX0 & $20 \mathrm{nkat} / \mathrm{L}$ \\
\hline \multicolumn{8}{|l|}{ Aldosterona (s) } \\
\hline dieta sódica normal & $8.1-15-5$ & $\mathrm{ng} / \mathrm{dL}$ & 27.74 & $220-430$ & $\mathrm{pmol} / \mathrm{L}$ & XX0 & $10 \mathrm{pmol} / \mathrm{L}$ \\
\hline dieta sódica restringida & $20.8-44.4$ & $\mathrm{ng} / \mathrm{dL}$ & 27.74 & $580-1240$ & $\mathrm{pmol} / \mathrm{L}$ & XX0 & $10 \mathrm{pmol} / \mathrm{L}$ \\
\hline \multicolumn{8}{|l|}{ Aldosterona (o)-excreción de sodio } \\
\hline$=25 \mathrm{mmol} / \mathrm{d}$ & $18-85$ & $\mu \mathrm{g} / 24 \mathrm{~h}$ & 2.774 & $50-235$ & $\mathrm{nmol} / \mathrm{d}$ & $\mathrm{XXX}$ & $5 \mathrm{nmol} / \mathrm{d}$ \\
\hline$=75-125 \mathrm{mmol} / \mathrm{d}$ & $5-26$ & $\mu \mathrm{g} / 24 \mathrm{~h}$ & 2.774 & $15-70$ & $\mathrm{nmol} / \mathrm{d}$ & $\mathrm{XXX}$ & $5 \mathrm{nmol} / \mathrm{L}$ \\
\hline$=200 \mathrm{mmol} / \mathrm{d}$ & $1.5-12.5$ & $\mu \mathrm{g} / 24 \mathrm{~h}$ & 2.774 & $5-35$ & $\mathrm{nmol} / \mathrm{L}$ & $\mathrm{XXX}$ & $5 \mathrm{nmol} / \mathrm{L}$ \\
\hline Fosfatasa alcalina (s) & $30-120$ & $\mathrm{U} / \mathrm{L}$ & 0.01667 & $0.5-2.0$ & $\mu \mathrm{kat} / \mathrm{L}$ & $\mathrm{X} . \mathrm{X}$ & $0.1 \mu \mathrm{kat} / \mathrm{L}$ \\
\hline Alfa-antitripsina (s) & $150-350$ & $\mathrm{ng} / \mathrm{dL}$ & 0.01 & $1.5-3.5$ & $\mathrm{~g} / \mathrm{L}$ & X.X & $0.1 \mathrm{~g} / \mathrm{L}$ \\
\hline Alfa-fetoproteína (s) & $0-20$ & $\mathrm{mg} / \mathrm{dL}$ & 1.00 & $0-20$ & $\mu \mathrm{g} / \mathrm{L}$ & $\mathrm{XX}$ & $1 \mu \mathrm{g} / \mathrm{L}$ \\
\hline Alfa-fetoproteína (LAm) & $\begin{array}{l}\text { Depende del } \\
\text { tiempo de } \\
\text { gestación }\end{array}$ & $\mathrm{Mg} / \mathrm{dL}$ & 10 & $\begin{array}{c}\text { Depende del } \\
\text { tiempo de } \\
\text { gestación }\end{array}$ & $\mathrm{mg} / \mathrm{L}$ & $\mathrm{XX}$ & $1 \mathrm{mg} / \mathrm{L}$ \\
\hline alfa $_{2}$-macroglobulina (s) & $145-510$ & $\mathrm{mg} / \mathrm{dL}$ & 0.01 & $1.5-4.1$ & $\mathrm{~g} / \mathrm{L}$ & $\mathrm{X} . \mathrm{X}$ & $0.1 \mathrm{~g} / \mathrm{L}$ \\
\hline aluminio (s) & $0-15$ & $\mathrm{ug} / \mathrm{L}$ & 37.06 & $0-560$ & $\mathrm{nmol} / \mathrm{L}$ & $\mathrm{XX0}$ & $10 \mathrm{nmol} / \mathrm{L}$ \\
\hline \multicolumn{8}{|l|}{ fracciones de aminoácidos $((\mathrm{P})$} \\
\hline alanina & $2.2-4.5$ & $\mathrm{mg} / \mathrm{dL}$ & 112.2 & $245-500$ & $\mu \mathrm{mol} / \mathrm{L}$ & XXX & $5 \mathrm{umol} / \mathrm{L}$ \\
\hline ácido alfa aminobutírico & $0.1-0.2$ & $\mathrm{mg} / \mathrm{dL}$ & 96.97 & $10-20$ & $\mu \mathrm{mol} / \mathrm{L}$ & $\mathrm{XXX}$ & $5 \mathrm{umol} / \mathrm{L}$ \\
\hline arginina & $0.5-2.5$ & $\mathrm{mg} / \mathrm{dL}$ & 57.40 & $30-145$ & $\mu \mathrm{mol} / \mathrm{L}$ & $\mathrm{XXX}$ & $5 \mathrm{umol} / \mathrm{L}$ \\
\hline asparagina & $0.5-0.5$ & $\mathrm{mg} / \mathrm{dL}$ & 75.69 & $35-45$ & $\mu \mathrm{mol} / \mathrm{L}$ & $\mathrm{XXX}$ & $5 \mathrm{umol} / \mathrm{L}$ \\
\hline ácido aspártico & $0.0-0.3$ & $\mathrm{mg} / \mathrm{dL}$ & 75.13 & $0-20$ & $\mu \mathrm{mol} / \mathrm{L}$ & $\mathrm{XXX}$ & $5 \mathrm{umol} / \mathrm{L}$ \\
\hline citrulina & $0.2-1.0$ & $\mathrm{mg} / \mathrm{dL}$ & 57.08 & $15-55$ & $\mu \mathrm{mol} / \mathrm{L}$ & $\mathrm{XXX}$ & $5 \mathrm{umol} / \mathrm{L}$ \\
\hline cistina & $0.2-2.2$ & $\mathrm{mg} / \mathrm{dL}$ & 41.61 & $10-90$ & $\mu \mathrm{mol} / \mathrm{L}$ & $\mathrm{XXX}$ & $5 \mathrm{umol} / \mathrm{L}$ \\
\hline ácido glutámico & $0.2-2.8$ & $\mathrm{mg} / \mathrm{dL}$ & 67.97 & $15-190$ & $\mu \mathrm{mol} / \mathrm{L}$ & $\mathrm{XXX}$ & $5 \mathrm{umol} / \mathrm{L}$ \\
\hline glutamina & $6.1-10.2$ & $\mathrm{mg} / \mathrm{dL}$ & 68.42 & $420-700$ & $\mu \mathrm{mol} / \mathrm{L}$ & $\mathrm{XXX}$ & $5 \mathrm{umol} / \mathrm{L}$ \\
\hline glicina & $0.9-4.2$ & $\mathrm{mg} / \mathrm{dL}$ & 133.2 & $120-560$ & $\mu \mathrm{mol} / \mathrm{L}$ & $\mathrm{XXX}$ & $5 \mathrm{umol} / \mathrm{L}$ \\
\hline histidina & $0.5-1.7$ & $\mathrm{mg} / \mathrm{dL}$ & 64.45 & $30-110$ & $\mu \mathrm{mol} / \mathrm{L}$ & $\mathrm{XXX}$ & $5 \mathrm{umol} / \mathrm{L}$ \\
\hline hidroxiprolina & 0 -trazas & $\mathrm{mg} / \mathrm{dL}$ & 76.26 & 0 -trazas & $\mu \mathrm{mol} / \mathrm{L}$ & $\mathrm{XXX}$ & $5 \mathrm{umol} / \mathrm{L}$ \\
\hline isoleucina & $0.5-1.3$ & $\mathrm{mg} / \mathrm{dL}$ & 76.24 & $40-100$ & $\mu \mathrm{mol} / \mathrm{L}$ & $\mathrm{XXX}$ & $5 \mathrm{umol} / \mathrm{L}$ \\
\hline leucina & $1.0-2.3$ & $\mathrm{mg} / \mathrm{dL}$ & 76.24 & $75-175$ & $\mu \mathrm{mol} / \mathrm{L}$ & $\mathrm{XXX}$ & $5 \mathrm{umol} / \mathrm{L}$ \\
\hline lisina & $2.2-3.5$ & $\mathrm{mg} / \mathrm{dL}$ & 68.40 & $80-240$ & $\mu \mathrm{mol} / \mathrm{L}$ & $\mathrm{XXX}$ & $5 \mathrm{umol} / \mathrm{L}$ \\
\hline metionina & $0.1-0.6$ & $\mathrm{mg} / \mathrm{dL}$ & 67.02 & $5-40$ & $\mu \mathrm{mol} / \mathrm{L}$ & $\mathrm{XXX}$ & $5 \mathrm{umol} / \mathrm{L}$ \\
\hline ornitina & $0.4-1.4$ & $\mathrm{mg} / \mathrm{dL}$ & 75.67 & $30-400$ & $\mu \mathrm{mol} / \mathrm{L}$ & $\mathrm{XXX}$ & $5 \mathrm{umol} / \mathrm{L}$ \\
\hline fenilalanina & $0.6-1.5$ & $\mathrm{mg} / \mathrm{dL}$ & 60.54 & $35-90$ & $\mu \mathrm{mol} / \mathrm{L}$ & $\mathrm{XXX}$ & $5 \mathrm{umol} / \mathrm{L}$ \\
\hline prolina & $1.2-3.9$ & $\mathrm{mg} / \mathrm{dL}$ & 86.86 & $105-340$ & $\mu \mathrm{mol} / \mathrm{L}$ & $\mathrm{XXX}$ & $5 \mu \mathrm{mol} / \mathrm{L}$ \\
\hline serina & $0.8-1.8$ & $\mathrm{mg} / \mathrm{dL}$ & 95.16 & $75-170$ & $\mu \mathrm{mol} / \mathrm{L}$ & $\mathrm{XXX}$ & $5 \mu \mathrm{mol} / \mathrm{L}$ \\
\hline taurina & $0.3-2.1$ & $\mathrm{mg} / \mathrm{dL}$ & 79.91 & $25-170$ & $\mu \mathrm{mol} / \mathrm{L}$ & $\mathrm{XXX}$ & $5 \mu \mathrm{mol} / \mathrm{L}$ \\
\hline treonina & $0.9-2.5$ & $\mathrm{mg} / \mathrm{dL}$ & 83.95 & $75-210$ & $\mu \mathrm{mol} / \mathrm{L}$ & $\mathrm{XXX}$ & $5 \mu \mathrm{mol} / \mathrm{L}$ \\
\hline triptófano & $0.5-2.5$ & $\mathrm{mg} / \mathrm{dL}$ & 48.97 & $25-125$ & $\mu \mathrm{mol} / \mathrm{L}$ & $\mathrm{XXX}$ & $5 \mu \mathrm{mol} / \mathrm{L}$ \\
\hline tirosina & $0.4-1.6$ & $\mathrm{mg} / \mathrm{dL}$ & 55.19 & $20-90$ & $\mu \mathrm{mol} / \mathrm{L}$ & $\mathrm{XXX}$ & $5 \mu \mathrm{mol} / \mathrm{L}$ \\
\hline valina & $1.7-3.7$ & $\mathrm{mg} / \mathrm{dL}$ & 85.36 & $145-315$ & $\mu \mathrm{mol} / \mathrm{L}$ & $\mathrm{XXX}$ & $5 \mu \mathrm{mol} / \mathrm{L}$ \\
\hline nitrógeno de aminoácido (P) & $4.0-6.0$ & $\mathrm{mg} / \mathrm{dL}$ & 0.7139 & $2.9-4.3$ & $\mathrm{mmol} / \mathrm{L}$ & X.X & $0.1 \mathrm{mmol} / \mathrm{L}$ \\
\hline nitrógeno de aminoácido (o) & $50-200$ & $\mathrm{mg} / 24 \mathrm{~h}$ & 0.07139 & $3.6-14.3$ & $\mathrm{mmol} / \mathrm{d}$ & X.X & $0.1 \mathrm{mmol} / \mathrm{d}$ \\
\hline $\begin{array}{l}\text { delta-aminoalevulinato (ácido levulinico) (o) } \\
\text { amitriptilina (P,s) }\end{array}$ & $1.0-7.0$ & $\mathrm{mg} / 24 \mathrm{~h}$ & 7.626 & $8-53$ & $\mu \mathrm{mol} / \mathrm{d}$ & $\mathrm{XX}$ & $1 \mu \mathrm{mol} / \mathrm{d}$ \\
\hline -terapéutico & $50-200$ & $\mathrm{ng} / \mathrm{mL}$ & 3.605 & $180-720$ & $\mathrm{nmol} / \mathrm{L}$ & XX0 & $10 \mathrm{nmol} / \mathrm{L}$ \\
\hline \multicolumn{8}{|l|}{ amoniaco (Pv) } \\
\hline como amoniaco $\left(\mathrm{NH}_{3}\right)$ & $10-80$ & $\mu \mathrm{g} / \mathrm{dL}$ & 0.5872 & $5-50$ & $\mu \mathrm{mol} / \mathrm{L}$ & $\mathrm{XXX}$ & $5 \mu \mathrm{mol} / \mathrm{L}$ \\
\hline como ion amonio $\left(\mathrm{NH}_{4}+\right)$ & $10-85$ & $\mu \mathrm{g} / \mathrm{dL}$ & 0.5543 & $5-50$ & $\mu \mathrm{mol} / \mathrm{L}$ & $\mathrm{XXX}$ & $5 \mu \mathrm{mol} / \mathrm{L}$ \\
\hline como nitrógeno $(\mathrm{N})$ & $10-65$ & $\mu \mathrm{g} / \mathrm{dL}$ & 0.7139 & $5-5$ & $\mu \mathrm{mol} / \mathrm{L}$ & $\mathrm{XXX}$ & $5 \mu \mathrm{mol} / \mathrm{L}$ \\
\hline amilasa (s) & $0-130$ & $\mathrm{U} / \mathrm{L}$ & 0.01667 & $0-2.17$ & $\mu \mathrm{kat} / \mathrm{L}$ & $\mathrm{XXX}$ & $0.01 \mathrm{ukat} / \mathrm{L}$ \\
\hline \multicolumn{8}{|l|}{ androstenediona (s) } \\
\hline -hombre mayor de 18 años & $0.2-3.0$ & $\mu \mathrm{g} / \mathrm{L}$ & 3.492 & $0.5-10.5$ & $\mathrm{nmol} / \mathrm{L}$ & XX.X & $0.5 \mathrm{nmol} / \mathrm{L}$ \\
\hline -mujer mayor de 18 años & $0.8-3-0$ & $\mu \mathrm{g} / \mathrm{L}$ & 3.492 & $3.0-10.5$ & $\mathrm{nmol} / \mathrm{L}$ & $\mathrm{XX} . \mathrm{X}$ & $0.5 \mathrm{nmol} / \mathrm{L}$ \\
\hline enzima convertidota de angiotensina (s) & $>40$ & $\mathrm{~mL} / \mathrm{min}$ & 16.62 & $>670$ & nkat/L & XX0 & $10 \mathrm{nkat} / \mathrm{L}$ \\
\hline
\end{tabular}


APENDICE

Tabla 2. (Continuación) Factores de conversión para los resultados de química clínica.

\begin{tabular}{|c|c|c|c|c|c|c|c|}
\hline Compuesto & $\begin{array}{l}\text { Intervalo de } \\
\text { referencia actual }\end{array}$ & $\begin{array}{l}\text { Unidad } \\
\text { actual }\end{array}$ & $\begin{array}{l}\text { Factores de } \\
\text { conversión }\end{array}$ & $\begin{array}{l}\text { Intervalos } \\
\text { de referencia } \\
\text { SI }\end{array}$ & $\begin{array}{l}\text { Símbolo de } \\
\text { unidad } \\
\text { SI }\end{array}$ & $\begin{array}{l}\text { Dígitos } \\
\text { significativos }\end{array}$ & $\begin{array}{l}\text { Mínimo } \\
\text { incremento } \\
\text { sugerido }\end{array}$ \\
\hline arsénico (C) & $>1$ & $\mu \mathrm{g} / \mathrm{g}(\mathrm{ppm})$ & 13.35 & $>13$ & $\mathrm{nmol} / \mathrm{g}$ & XX.X & $0.5 \mathrm{nmol} / \mathrm{g}$ \\
\hline arsénico (o) & $0-5$ & $\mu \mathrm{g} / 24 \mathrm{~h}$ & 13.35 & $0-67$ & $\mathrm{nmol} / \mathrm{d}$ & XX & $1 \mathrm{nmol} / \mathrm{d}$ \\
\hline ascorbato (P) (ácido ascórbico) & $0.6-2.0$ & $\mathrm{mg} / \mathrm{dL}$ & 56.78 & $30-110$ & $\mu \mathrm{mol} / \mathrm{L}$ & $\mathrm{X} 0$ & $10 \mu \mathrm{mol} / \mathrm{L}$ \\
\hline aspartato aminotransferasa (s) & $0-35$ & $\mathrm{U} / \mathrm{L}$ & 0.01667 & $0-0.58$ & $\mu \mathrm{kat} / \mathrm{L}$ & $0 . \mathrm{XX}$ & $0.01 \mathrm{ukat} / \mathrm{L}$ \\
\hline \multirow{2}{*}{$\begin{array}{l}\text { barbiturato (s)-sobredosis } \\
\text { el total expresado como: }\end{array}$} & & & & & & & \\
\hline & $\begin{array}{l}\text { depende de la } \\
\text { composición } \\
\text { de la mezcla }\end{array}$ & & & & & & \\
\hline fenobarbital & $\ldots \ldots$ & $\mathrm{mg} / \mathrm{dL}$ & 43.06 & $\ldots \ldots$ & $\mu \mathrm{mol} / \mathrm{L}$ & $\mathrm{XX}$ & $5 \mu \mathrm{mol} / \mathrm{L}$ \\
\hline fenobarbital sódico & $\ldots \ldots$. & $\mathrm{mg} / \mathrm{dL}$ & 39.34 & $\ldots \ldots \ldots$ & $\mu \mathrm{mol} / \mathrm{L}$ & $\mathrm{XX}$ & $5 \mu \mathrm{mol} / \mathrm{L}$ \\
\hline barbitona & $\ldots \ldots \ldots$ & $\mathrm{mg} / \mathrm{dL}$ & 54.29 & ......... & $\mu \mathrm{mol} / \mathrm{L}$ & $\mathrm{XX}$ & $5 \mu \mathrm{mol} / \mathrm{L}$ \\
\hline \multicolumn{8}{|l|}{ barbiturato (s)-terapéutico } \\
\hline ver fenobarbital & ….... & …...... & …..... & …..... & …..... & …..... & …..... \\
\hline ver pentobarvital & $\ldots \ldots \ldots$ & .......... & ........... & ........... & .......... & .......... & ........... \\
\hline ver tiopental & $\ldots \ldots \ldots$ & .......... & .......... & ........... & .......... & …...... & .......... \\
\hline \multicolumn{8}{|l|}{ ácidos biliares, total (s) } \\
\hline (como ácido quenodeoxicólico) & trazas-3.3 & $\mu \mathrm{g} / \mathrm{mL}$ & 2.547 & trazas- 8.4 & $\mu \mathrm{mol} / \mathrm{L}$ & X.X & $0.2 \mu \mathrm{mol} / \mathrm{L}$ \\
\hline ácido cólico & trazas-1.0 & $\mu \mathrm{g} / \mathrm{mL}$ & 2.448 & trazas-2.4 & $\mu \mathrm{mol} / \mathrm{L}$ & X.X & $0.2 \mu \mathrm{mol} / \mathrm{L}$ \\
\hline ácido quenodeoxicólico & trazas-1.3 & $\mu \mathrm{g} / \mathrm{mL}$ & 2.547 & trazas-3.4 & $\mu \mathrm{mol} / \mathrm{L}$ & X.X & $0.2 \mu \mathrm{mol} / \mathrm{L}$ \\
\hline ácido deoxicólico & trazas-1.0 & $\mu \mathrm{g} / \mathrm{mL}$ & 2.547 & trazas- 2.5 & $\mu \mathrm{mol} / \mathrm{L}$ & X.X & $0.2 \mu \mathrm{mol} / \mathrm{L}$ \\
\hline ácido licrocólico & trazas & $\mu \mathrm{g} / \mathrm{mL}$ & 5.656 & trazas & $\mu \mathrm{mol} / \mathrm{L}$ & X.X & $0.2 \mu \mathrm{mol} / \mathrm{L}$ \\
\hline \multicolumn{8}{|l|}{ ácidos biliares (líquido duodenal) } \\
\hline \multicolumn{8}{|l|}{ (después de estímulo con colecistoquinina) } \\
\hline & & & & & & & \\
\hline ácido & $14.0-58.0$ & $\mathrm{mg} / \mathrm{mL}$ & 2.547 & $35.0-148.0$ & $\mathrm{mmol} / \mathrm{L}$ & $\mathrm{XX} . \mathrm{X}$ & $0.2 \mathrm{mmol} / \mathrm{L}$ \\
\hline ácido cólico & $2.4-33.0$ & $\mathrm{mg} / \mathrm{mL}$ & 2.448 & $6.8-81.0$ & $\mathrm{mmol} / \mathrm{L}$ & XX.X & $0.2 \mathrm{mmol} / \mathrm{L}$ \\
\hline ácido quenodeoxicólico & $4.0-24.0$ & $\mathrm{mg} / \mathrm{mL}$ & 2.547 & $10.0-61.4$ & $\mathrm{mmol} / \mathrm{L}$ & XX.X & $0.2 \mathrm{mmol} / \mathrm{L}$ \\
\hline ácido deoxicólico & $0.8-6.9$ & $\mathrm{mg} / \mathrm{mL}$ & 2.547 & $2.0-18.0$ & $\mathrm{mmol} / \mathrm{L}$ & XX.X & $0.2 \mathrm{mmol} / \mathrm{L}$ \\
\hline ácido litocólico & $0.3-0.8$ & $\mathrm{mg} / \mathrm{mL}$ & 2.656 & $0.8-2.0$ & $\mathrm{mmol} / \mathrm{L}$ & $\mathrm{XX} . \mathrm{X}$ & $0.2 \mathrm{mmol} / \mathrm{L}$ \\
\hline bilirrubina, total (s) & $0.1-1.0$ & $\mathrm{mg} / \mathrm{dL}$ & 17.10 & 2.18 & $\mu \mathrm{mol} / \mathrm{L}$ & $\mathrm{XX}$ & $2 \mu \mathrm{ml} / \mathrm{L}$ \\
\hline bilirrubina, conjugada (s) & & $\mathrm{mg} / \mathrm{dL}$ & 17.10 & $0-4$ & $\mu \mathrm{mol} / \mathrm{L}$ & $\mathrm{XX}$ & $2 \mu \mathrm{mol} / \mathrm{L}$ \\
\hline \multicolumn{8}{|l|}{ bromuros (s), tóxico } \\
\hline -como ion bromuro & $>120$ & $\mathrm{mg} / \mathrm{dL}$ & 0.1252 & $>15$ & $\mathrm{mmol} / \mathrm{L}$ & $\mathrm{XX}$ & $1 \mathrm{mmol} / \mathrm{L}$ \\
\hline -como bromuro de sodio & $>150$ & $\mathrm{mg} / \mathrm{dL}$ & 0.09719 & $>15$ & $\mathrm{mmol} / \mathrm{L}$ & $\mathrm{XX}$ & $1 \mathrm{mmol} / \mathrm{L}$ \\
\hline & $>15$ & $\mathrm{mEq} / \mathrm{L}$ & 1.00 & $>15$ & $\mathrm{mmol} / \mathrm{L}$ & $\mathrm{XX}$ & $1 \mathrm{mmol} / \mathrm{L}$ \\
\hline cadmio (s) & $<3$ & $\mathrm{ug} / \mathrm{dL}$ & 0.08897 & $<0.3$ & $\mu \mathrm{mol} / \mathrm{L}$ & X.X & $0.1 \mu \mathrm{mol} / \mathrm{L}$ \\
\hline calcitonina (s) & $<100$ & $\mathrm{pg} / \mathrm{mL}$ & 1.00 & $<100$ & $\mathrm{ng} / \mathrm{L}$ & $\mathrm{XXX}$ & $10 \mathrm{ng} / \mathrm{L}$ \\
\hline \multicolumn{8}{|l|}{ calcio (s) } \\
\hline -hombre & $8.8-10.3$ & $\mathrm{mg} / \mathrm{dL}$ & 0.2495 & $2.20-2.58$ & $\mathrm{mmol} / \mathrm{L}$ & $\mathrm{X} . \mathrm{XX}$ & $0.02 \mathrm{mmol} / \mathrm{L}$ \\
\hline -mujer menor de 50 años & $8.8-10.0$ & $\mathrm{mg} / \mathrm{dL}$ & 0.2495 & $2.20-2.50$ & $\mathrm{mmol} / \mathrm{L}$ & $\mathrm{X} . \mathrm{XX}$ & $0.02 \mathrm{mmol} / \mathrm{L}$ \\
\hline \multirow[t]{2}{*}{-mujer mayor de 50 años } & $8.8-10.2$ & $\mathrm{mg} / \mathrm{dL}$ & 0.2495 & $2.20-2.56$ & $\mathrm{mmol} / \mathrm{L}$ & $\mathrm{X} . \mathrm{XX}$ & $0.02 \mathrm{mmol} / \mathrm{L}$ \\
\hline & 4.4-5.1 & $\mathrm{mEq} / \mathrm{L}$ & 0.500 & $2.20-2.56$ & $\mathrm{mmol} / \mathrm{L}$ & $\mathrm{X} . \mathrm{XX}$ & $0.02 \mathrm{mmol} / \mathrm{L}$ \\
\hline \multirow[t]{2}{*}{ calcio iónico (s) } & $2.00-2.30$ & $\mathrm{mEq} / \mathrm{L}$ & 0.500 & $1.00-1.15$ & $\mathrm{mmol} / \mathrm{L}$ & X.XX & $0.01 \mathrm{mmol} / \mathrm{L}$ \\
\hline & $4.00-4.60$ & $\mathrm{mg} / \mathrm{dL}$ & 0.2495 & $1.00-1.15$ & $\mathrm{mmol} / \mathrm{L}$ & $\mathrm{X} . \mathrm{XX}$ & $0.01 \mathrm{mmol} / \mathrm{L}$ \\
\hline calcio (o), dieta normal & $<250$ & $\mathrm{mg} / 24 \mathrm{~h}$ & 0.02495 & $<6.2$ & $\mathrm{mmol} / \mathrm{d}$ & X.X & $0.1 \mathrm{mmol} / \mathrm{L}$ \\
\hline carbamacepina (P)-terapéutico & $4.0-10.0$ & $\mathrm{mg} / \mathrm{L}$ & 4.233 & $17-42$ & $\mu \mathrm{mol} / \mathrm{d}$ & $\mathrm{XX}$ & $1 \mu \mathrm{mol} / \mathrm{L}$ \\
\hline \multicolumn{8}{|l|}{ contenido de dióxido de carbono } \\
\hline$(\mathrm{S}, \mathrm{P}, \mathrm{s})\left(\right.$ bicarbonato $\left.+\mathrm{CO}_{2}\right)$ & $22-28$ & $\mathrm{mEq} / \mathrm{L}$ & 1.00 & $22-28$ & $\mathrm{mmol} / \mathrm{L}$ & $\mathrm{XX}$ & $1 \mathrm{mmol} / \mathrm{L}$ \\
\hline \multicolumn{8}{|l|}{ monóxido de carbono (S) } \\
\hline (proporción de $\mathrm{Hb}$ en forma de $\mathrm{COHb}$ ) & $<15$ & $\%$ & 0.01 & $<0.15$ & 1 & $0 . \mathrm{XX}$ & 0.01 \\
\hline beta-carotenos (s) & $50-250$ & $\mu \mathrm{g} / \mathrm{dL}$ & 0.01863 & $0.9-4.6$ & $\mu \mathrm{mol} / \mathrm{L}$ & $\mathrm{X} . \mathrm{X}$ & $0.1 \mu \mathrm{mol} / \mathrm{L}$ \\
\hline \multicolumn{8}{|l|}{ catecolaminas (o) } \\
\hline (como norepirefrina) & $<120$ & $\mu \mathrm{g} / 24 \mathrm{~h}$ & 5.911 & $<675$ & $\mathrm{nmol} / \mathrm{d}$ & XX0 & $10 \mathrm{mg} / \mathrm{d}$ \\
\hline ceruloplasmina (s) & $20-35$ & $\mathrm{mg} / \mathrm{dL}$ & 10.0 & $200-350$ & $\mathrm{mg} / \mathrm{L}$ & XX0 & $10 \mathrm{mg} / \mathrm{L}$ \\
\hline \multicolumn{8}{|l|}{ clordiazepóxido (P) } \\
\hline -terapéutico & $0.5-5.0$ & $\mathrm{mg} / \mathrm{L}$ & 3.336 & $2-17$ & $\mu \mathrm{mol} / \mathrm{L}$ & $\mathrm{XX}$ & $1 \mu \mathrm{mol} / \mathrm{L}$ \\
\hline -tóxico & $>10.0$ & $\mathrm{mg} / \mathrm{L}$ & 3.336 & $>33$ & $\mu \mathrm{mol} / \mathrm{L}$ & $\mathrm{XX}$ & $1 \mu \mathrm{mol} / \mathrm{L}$ \\
\hline cloruros $(\mathrm{s})$ & $95-105$ & $\mathrm{mEq} / \mathrm{L}$ & 1.00 & $95-105$ & $\mathrm{mmol} / \mathrm{L}$ & $\mathrm{XXX}$ & $1 \mathrm{mmol} / \mathrm{L}$ \\
\hline clorimipramina $(\mathrm{P})$ & $50-400$ & $\mathrm{ng} / \mathrm{mL}$ & 3.176 & $150-1270$ & $\mathrm{nmol} / \mathrm{L}$ & XX0 & $10 \mathrm{nmol} / \mathrm{L}$ \\
\hline clorpromazina (P) & $50-300$ & $\mathrm{ng} / \mathrm{mL}$ & 3.136 & $150-950$ & $\mathrm{nmol} / \mathrm{L}$ & $\mathrm{XX0}$ & $10 \mathrm{nmol} / \mathrm{L}$ \\
\hline \multicolumn{8}{|l|}{ clorprofamida $(\mathrm{P})$} \\
\hline -terapéutica & $75-250$ & $\mathrm{mg} / \mathrm{L}$ & 3.613 & $270-900$ & $\mu \mathrm{mol} / \mathrm{L}$ & XX0 & $10 \mu \mathrm{mol} / \mathrm{L}$ \\
\hline colestanol (P)-(como fracción del colesterol total) & $1-3$ & $\%$ & 0.01 & $0.01-0.03$ & 1 & $0 . \mathrm{XX}$ & 0.01 \\
\hline
\end{tabular}


APENDICE

Tabla 2. (Continuación) Factores de conversión para los resultados de química clínica.

\begin{tabular}{|c|c|c|c|c|c|c|c|}
\hline Compuesto & $\begin{array}{c}\text { Intervalo de } \\
\text { referencia actual }\end{array}$ & $\begin{array}{l}\text { Unidad } \\
\text { actual }\end{array}$ & $\begin{array}{l}\text { Factores de } \\
\text { conversión }\end{array}$ & $\begin{array}{l}\text { Intervalos } \\
\text { de referencia } \\
\text { SI }\end{array}$ & $\begin{array}{l}\text { Símbolo de } \\
\text { unidad } \\
\text { SI }\end{array}$ & $\begin{array}{c}\text { Dígitos } \\
\text { significativos }\end{array}$ & $\begin{array}{l}\text { Mínimo } \\
\text { incremento } \\
\text { sugerido }\end{array}$ \\
\hline \multicolumn{8}{|l|}{ colesterol (P) } \\
\hline -menor de 29 años & $<200$ & $\mathrm{mg} / \mathrm{dL}$ & 0.02586 & $<5.20$ & $\mathrm{mmol} / \mathrm{L}$ & X.XX & $0.05 \mathrm{mmol} / \mathrm{L}$ \\
\hline$-30-39$ años & $<225$ & $\mathrm{mg} / \mathrm{dL}$ & 0.02586 & $<5.85$ & $\mathrm{mmol} / \mathrm{L}$ & X.XX & $0.05 \mathrm{mmol} / \mathrm{L}$ \\
\hline$-40-49$ años & $<245$ & $\mathrm{mg} / \mathrm{dL}$ & 0.02586 & $<6.35$ & $\mathrm{mmol} / \mathrm{L}$ & X.XX & $0.05 \mathrm{mmol} / \mathrm{L}$ \\
\hline -mayor de 50 años & $<265$ & $\mathrm{mg} / \mathrm{dL}$ & 0.02586 & $<6.85$ & $\mathrm{mmol} / \mathrm{L}$ & X.XX & $0.05 \mathrm{mmol} / \mathrm{L}$ \\
\hline \multicolumn{8}{|l|}{ ésteres del colesterol (P) (como fracción } \\
\hline del colesterol total) & $60-75$ & $\%$ & 0.01 & $0.60-0.75$ & 1 & $0 . \mathrm{XX}$ & 0.01 \\
\hline colinesteresa (s) & $620-1370$ & $\mathrm{U} / \mathrm{L}$ & 0.01667 & $10.3-22.8$ & $\mu \mathrm{kat} / \mathrm{L}$ & XX.X & $0.1 \mu \mathrm{kat} / \mathrm{L}$ \\
\hline \multicolumn{8}{|l|}{ gonadotropina coriónica (P) (beta-HCG) } \\
\hline citrato (S) (ácido cítrico) & $1.2-3.0$ & $\mathrm{mg} / \mathrm{dL}$ & 52.05 & $60-160$ & $\mu \mathrm{mol} / \mathrm{L}$ & $\mathrm{XXX}$ & $5 \mu \mathrm{mol} / \mathrm{L}$ \\
\hline complemento, $\mathrm{C}_{3}(\mathrm{~s})$ & $70-160$ & $\mathrm{mg} / \mathrm{dL}$ & 0.01 & $0.7-1.6$ & $\mathrm{~g} / \mathrm{L}$ & $\mathrm{X} . \mathrm{X}$ & $0.1 \mathrm{~g} / \mathrm{L}$ \\
\hline complemento, $\mathrm{C}_{4}(\mathrm{~s})$ & $20-40$ & $\mathrm{mg} / \mathrm{dL}$ & 0.01 & $0.2-0.4$ & $\mathrm{~g} / \mathrm{L}$ & X.X & $0.1 \mathrm{~g} / \mathrm{L}$ \\
\hline cobre (s) & $70-140$ & $\mu \mathrm{g} / \mathrm{dL}$ & 0.1574 & $11.0-22.0$ & $\mu \mathrm{mol} / \mathrm{L}$ & XX.X & $0.2 \mu \mathrm{mol} / \mathrm{L}$ \\
\hline cobre (o) & $<40$ & $\mu \mathrm{g} / 24 \mathrm{~h}$ & 0.01574 & $<0.6$ & $\mu \mathrm{mol} / \mathrm{d}$ & X.X & $0.2 \mu \mathrm{mol} / \mathrm{d}$ \\
\hline coproporfirinas (o) & $<200$ & $\mu \mathrm{g} / 24 \mathrm{~h}$ & 1.527 & $<300$ & $\mathrm{nmol} / \mathrm{d}$ & XX0 & $10 \mathrm{nmol} / \mathrm{d}$ \\
\hline \multicolumn{8}{|l|}{ cortisol (s) } \\
\hline-0800 horas & $4-19$ & $\mu \mathrm{g} / \mathrm{dL}$ & 27.59 & $110-520$ & $\mathrm{nmol} / \mathrm{L}$ & XX0 & $10 \mathrm{nmol} / \mathrm{L}$ \\
\hline-1600 horas & $2-15$ & $\mu \mathrm{g} / \mathrm{dL}$ & 27.59 & $50-410$ & $\mathrm{nmol} / \mathrm{L}$ & XX0 & $10 \mathrm{nmol} / \mathrm{L}$ \\
\hline-2400 horas & 5 & $\mu \mathrm{g} / \mathrm{dL}$ & 27.59 & 140 & $\mathrm{nmol} / \mathrm{L}$ & XX0 & $10 \mathrm{nmol} / \mathrm{L}$ \\
\hline cortisol libre (o) & $10-110$ & $\mu \mathrm{g} / 24 \mathrm{~h}$ & 27.59 & $30-300$ & $\mathrm{nmol} / \mathrm{d}$ & XX0 & $10 \mathrm{nmol} / \mathrm{d}$ \\
\hline \multicolumn{8}{|l|}{ creatina (s) } \\
\hline -hombre & $0.17-0.50$ & $\mathrm{mg} / \mathrm{dL}$ & 76.25 & $10-40$ & $\mu \mathrm{mol} / \mathrm{L}$ & $\mathrm{X} 0$ & $10 \mu \mathrm{mol} / \mathrm{L}$ \\
\hline -mujer & $0.35-0.93$ & $\mathrm{mg} / \mathrm{dL}$ & 76.25 & $30-70$ & $\mu \mathrm{mol} / \mathrm{L}$ & $\mathrm{X} 0$ & $10 \mu \mathrm{mol} / \mathrm{L}$ \\
\hline \multicolumn{8}{|l|}{ cretina (o) } \\
\hline -hombre & $0-40$ & $\mathrm{mg} / 24 \mathrm{~h}$ & 7.625 & $0-300$ & $\mu \mathrm{mol} / \mathrm{d}$ & XX0 & $10 \mu \mathrm{mol} / \mathrm{d}$ \\
\hline -mujer & $0-80$ & $\mathrm{mg} / 24 \mathrm{~h}$ & 7.625 & $0-600$ & $\mu \mathrm{mol} / \mathrm{d}$ & $\mathrm{XX0}$ & $10 \mu \mathrm{mol} / \mathrm{d}$ \\
\hline \multicolumn{8}{|l|}{ creatin quinasa (CK) (s) } \\
\hline isoenzimas de creatin quinasa (s) & $0-130$ & $\mathrm{U} / \mathrm{L}$ & 0.01667 & $0-2.16$ & $\mu \mathrm{kat} / \mathrm{L}$ & X.XX & $0.1 \mu \mathrm{kat} / \mathrm{L}$ \\
\hline \multirow{2}{*}{$\begin{array}{l}\text { fracción de MB } \\
\text { creatinina (s) }\end{array}$} & $>5$ & $\%$ & 0.01 & 0.05 & 1 & $0 . \mathrm{XX}$ & 0.01 \\
\hline & $0.6-1.2$ & $\mathrm{mg} / \mathrm{dL}$ & 88.40 & $50-110$ & $\mu \mathrm{mol} / \mathrm{L}$ & XX0 & $10 \mu \mathrm{mol} / \mathrm{L}$ \\
\hline creatinina $(o)$ & Variable & $\mathrm{g} / 24 \mathrm{~h}$ & 8.840 & Variable & $\mathrm{mmol} / \mathrm{d}$ & XX.X & $0.1 \mathrm{mmol} / \mathrm{d}$ \\
\hline depuración de creatinina $(\mathrm{s}, \mathrm{o})$ & $75-125$ & & & $\mathrm{~mL} / \mathrm{min}$ & 0.1667 & $1.24-2.08$ & $\mathrm{~mL} / \mathrm{s}$ \\
\hline \multirow[t]{2}{*}{$\mathrm{X} . \mathrm{XX}$} & $0.2 \mathrm{~mL} / \mathrm{s}$ & & & & & & \\
\hline & \multicolumn{7}{|c|}{ depuración de creatinina $=1 \mu \mathrm{mol} / \mathrm{L}$ (creatinina urinaria) $\times \mathrm{mL} / \mathrm{s} \times 1.73$} \\
\hline cianuro (s) - letal & 0.10 & $\mathrm{mg} / \mathrm{dL}$ & 384.3 & 40 & $\mu \mathrm{mol} / \mathrm{L}$ & $\mathrm{XXX}$ & $5 \mu \mathrm{mol} / \mathrm{L}$ \\
\hline cianocobalamina (s) & & & & & & & \\
\hline$\left(\right.$ vitamina $\left.\mathrm{B}_{12}\right)$ & $200-100$ & $\mathrm{pg} / \mathrm{mL}$ & 0.7378 & $150-750$ & $\mathrm{pmol} / \mathrm{L}$ & XX.0 & $10 \mathrm{pmol} / \mathrm{L}$ \\
\hline AMP cíclico (s) & $2.6-6.6$ & $\mu \mathrm{g} / \mathrm{L}$ & 3.038 & $9-20$ & $\mathrm{nmol} / \mathrm{L}$ & XXX & $1 \mathrm{nmol} / \mathrm{L}$ \\
\hline \multicolumn{8}{|l|}{ AMP cíclico (o) } \\
\hline uranio total & $2.9-5.6$ & $\begin{array}{c}\mu \mathrm{mol} / \mathrm{g} \\
\text { creatinina }\end{array}$ & 113.1 & $330-630$ & $\begin{array}{l}\mathrm{nmol} / \mathrm{mmol} \\
\text { creatinina }\end{array}$ & XX.0 & $\begin{array}{c}10 \mathrm{nmol} / \mathrm{mmol} \\
\text { creatinina }\end{array}$ \\
\hline tubular renal & $<2.5$ & $\begin{array}{c}\mu \mathrm{mol} / \mathrm{g} \\
\text { creatinina }\end{array}$ & 113.1 & $<280$ & $\begin{array}{l}\mathrm{nmol} / \mathrm{mmol} \\
\text { creatinina }\end{array}$ & XX.0 & $\begin{array}{c}10 \mathrm{nmol} / \mathrm{mmol} \\
\text { creatinina }\end{array}$ \\
\hline GMP cíclico (s) & $0.6-3.5$ & $\mu \mathrm{g} / \mathrm{L}$ & 2.897 & $1.7-10.1$ & $\mathrm{nmol} / \mathrm{L}$ & XX.X & $10 \mathrm{nmol} / \mathrm{L}$ \\
\hline GMP cíclico (o) & $0.3-1.8$ & $\begin{array}{c}\mu \mathrm{mol} / \mathrm{g} \\
\text { creatinina }\end{array}$ & 113.1 & $30-200$ & $\begin{array}{l}\mathrm{nmol} / \mathrm{mmol} \\
\text { creatinina }\end{array}$ & XX0 & $\begin{array}{c}10 \mathrm{nmol} / \mathrm{mmol} \\
\text { creatinina }\end{array}$ \\
\hline cistina (o) & $10-100$ & $\mathrm{mg} / 24 \mathrm{~h}$ & 4.161 & $40-420$ & $\mu \mathrm{mol} / \mathrm{d}$ & XX0 & $10 \mu \mathrm{mol} / \mathrm{d}$ \\
\hline \multicolumn{8}{|l|}{ dehidroespiandrosterona $(\mathrm{P}, \mathrm{s})$} \\
\hline (DHEA) - 1-4 años & $0.2-0.4$ & $\mu \mathrm{g} / \mathrm{L}$ & 3.467 & $0.6-1.4$ & $\mathrm{nmol} / \mathrm{L}$ & XX.X & $0.2 \mathrm{nmol} / \mathrm{L}$ \\
\hline 4-8 años & $0.1-1.9$ & $\mu \mathrm{g} / \mathrm{L}$ & 3.467 & $0.4-6.6$ & $\mathrm{nmol} / \mathrm{L}$ & XX.X & $0.2 \mathrm{nmol} / \mathrm{L}$ \\
\hline 8-10 años & $0.2-2.9$ & $\mu \mathrm{g} / \mathrm{L}$ & 3.467 & $0.6-10.0$ & $\mathrm{nmol} / \mathrm{L}$ & XX.X & $0.2 \mathrm{nmol} / \mathrm{L}$ \\
\hline 10-12 años & $0.5-9.2$ & $\mu \mathrm{g} / \mathrm{L}$ & 3.467 & $1.8-31.8$ & $\mathrm{nmol} / \mathrm{L}$ & XX.X & $0.2 \mathrm{nmol} / \mathrm{L}$ \\
\hline 12-14 años & $0.9-20.0$ & $\mu \mathrm{g} / \mathrm{L}$ & 3.467 & $3.2-69.4$ & $\mathrm{nmol} / \mathrm{L}$ & XX.X & $0.2 \mathrm{nmol} / \mathrm{L}$ \\
\hline 14-16 años & $2.5-20.0$ & $\mu \mathrm{g} / \mathrm{L}$ & 3.467 & $8.6-69.4$ & $\mathrm{nmol} / \mathrm{L}$ & XX.X & $0.2 \mathrm{nmol} / \mathrm{L}$ \\
\hline mujer premenopáusica & $2.0-15.0$ & $\mu \mathrm{g} / \mathrm{L}$ & 3.467 & $7.0-52.0$ & $\mathrm{nmol} / \mathrm{L}$ & XX.X & $0.2 \mathrm{nmol} / \mathrm{L}$ \\
\hline hombre & $0.8-10.0$ & $\mu \mathrm{g} / \mathrm{L}$ & 3.467 & $2.8-34.6$ & $\mathrm{nmol} / \mathrm{L}$ & XX.X & $0.2 \mathrm{nmol} / \mathrm{L}$ \\
\hline dehidroepiandosterona (o) & $\begin{array}{l}\text { ver fraccionamiento } \\
\text { de esteroides }\end{array}$ & & & & & & \\
\hline
\end{tabular}




\section{APENDICE}

Tabla 2. (Continuación) Factores de conversión para los resultados de química clínica.

\begin{tabular}{|c|c|c|c|c|c|c|c|}
\hline Compuesto & $\begin{array}{l}\text { Intervalo de } \\
\text { referencia actual }\end{array}$ & $\begin{array}{l}\text { Unidad } \\
\text { actual }\end{array}$ & $\begin{array}{l}\text { Factores de } \\
\text { conversión }\end{array}$ & $\begin{array}{l}\text { Intervalos } \\
\text { de referencia } \\
\text { SI }\end{array}$ & $\begin{array}{l}\text { Símbolo de } \\
\text { unidad } \\
\text { SI }\end{array}$ & $\begin{array}{c}\text { Dígitos } \\
\text { significativos }\end{array}$ & $\begin{array}{l}\text { Mínimo } \\
\text { incremento } \\
\text { sugerido }\end{array}$ \\
\hline \multicolumn{8}{|l|}{$\begin{array}{l}\text { dehidroepiandosterona } \\
\text { (sulfato) } \\
\text { (P,s) }\end{array}$} \\
\hline recién nacido & $1670-3640$ & $\mathrm{ng} / \mathrm{mL}$ & 0.002714 & $4.5-9.9$ & $\mu \mathrm{mol} / \mathrm{L}$ & XX.X & $0.1 \mu \mathrm{mol} / \mathrm{L}$ \\
\hline niños pre-puberales & $100-600$ & $\mathrm{ng} / \mathrm{mL}$ & 0.002714 & $0.3-1.6$ & $\mu \mathrm{mol} / \mathrm{L}$ & XX.X & $0.1 \mu \mathrm{mol} / \mathrm{L}$ \\
\hline hombre & $2000-3350$ & $\mathrm{ng} / \mathrm{mL}$ & 0.002714 & $5.4-9.1$ & $\mu \mathrm{mol} / \mathrm{L}$ & XX.X & $0.1 \mu \mathrm{mol} / \mathrm{L}$ \\
\hline mujer (premenopáusica) & $820-3380$ & $\mathrm{ng} / \mathrm{mL}$ & 0.002714 & $2.2-9.2$ & $\mu \mathrm{mol} / \mathrm{L}$ & XX.X & $0.1 \mu \mathrm{mol} / \mathrm{L}$ \\
\hline mujer (post-menopáusica) & $110-610$ & $\mathrm{ng} / \mathrm{mL}$ & 0.002714 & $0.3-1.7$ & $\mu \mathrm{mol} / \mathrm{L}$ & XX.X & $0.1 \mu \mathrm{mol} / \mathrm{L}$ \\
\hline embarazo a término & $230-1170$ & $\mathrm{ng} / \mathrm{mL}$ & 0.002714 & $0.6-3.2$ & $\mu \mathrm{mol} / \mathrm{L}$ & XX.X & $0.1 \mu \mathrm{mol} / \mathrm{L}$ \\
\hline 11-deoxicordisol (s) & $0-2$ & $\mu \mathrm{g} / \mathrm{dL}$ & 28.86 & $0-60$ & $\mathrm{nmol} / \mathrm{L}$ & XX0 & $10 \mathrm{nmol} / \mathrm{L}$ \\
\hline $\begin{array}{l}\text { desipramına }(\mathrm{P}) \\
\text { terapéutico } \\
\text { diazepam }(\mathrm{P})\end{array}$ & $50-200$ & $\mathrm{ng} / \mathrm{mL}$ & 3.754 & $170-700$ & $\mathrm{nmol} / \mathrm{L}$ & XX0 & $10 \mathrm{nmol} / \mathrm{L}$ \\
\hline -terapéutico & $0.10-0.25$ & $\mathrm{mg} / \mathrm{L}$ & 3512 & $350-900$ & $\mathrm{nmol} / \mathrm{L}$ & XX0 & $10 \mathrm{nmol} / \mathrm{L}$ \\
\hline -tóxico & $<1.0$ & $\mathrm{mg} / \mathrm{L}$ & 3512 & $>3510$ & $\mathrm{nmol} / \mathrm{L}$ & XX0 & $10 \mathrm{nmol} / \mathrm{L}$ \\
\hline $\begin{array}{l}\text {-terapéutico } \\
\text { digoxina }(\mathrm{P})\end{array}$ & $8-30$ & $\mathrm{mg} / \mathrm{L}$ & 2.974 & $25-90$ & $\mu \mathrm{mol} / \mathrm{L}$ & $\mathrm{XX}$ & $5 \mu \mathrm{mol} / \mathrm{L}$ \\
\hline -terapéutico & $\begin{array}{l}0.5-2.2 \\
0.5-2.2\end{array}$ & $\begin{array}{c}\mathrm{ng} / \mathrm{mL} \\
\mu \mathrm{g} / \mathrm{L}\end{array}$ & $\begin{array}{l}1.281 \\
1.281\end{array}$ & $\begin{array}{l}0.6-2.8 \\
0.6-2.8\end{array}$ & $\begin{array}{l}\mathrm{nmol} / \mathrm{L} \\
\mathrm{nmol} / \mathrm{L}\end{array}$ & $\begin{array}{l}X . X \\
X . X\end{array}$ & $\begin{array}{l}0.1 \mathrm{nmol} / \mathrm{L} \\
0.1 \mathrm{nmol} / \mathrm{L}\end{array}$ \\
\hline \multicolumn{8}{|l|}{ dimetadiona $(\mathrm{P})$} \\
\hline $\begin{array}{l}\text {-terapéutico } \\
\text { disopiramida }(\mathrm{P})\end{array}$ & $<1.00$ & $g / L$ & 7.745 & $<7.7$ & $\mathrm{mmol} / \mathrm{L}$ & X.X & $0.1 \mathrm{mmol} / \mathrm{L}$ \\
\hline $\begin{array}{l}\text {-terapéutico } \\
\text { doxepina }(\mathrm{P})\end{array}$ & $2.0-6.0$ & $\mathrm{mg} / \mathrm{L}$ & 2.946 & $6-18$ & $\mu \mathrm{mol} / \mathrm{L}$ & $\mathrm{XX}$ & $1 \mu \mathrm{mol} / \mathrm{L}$ \\
\hline \multicolumn{7}{|l|}{ electroforesis de proteínas (s) } & $10 \mathrm{nmol} / \mathrm{L}$ \\
\hline albúmina & $60-.65$ & $\%$ & 0.01 & $0.60-0.65$ & 1 & $0 . \mathrm{XX}$ & 0.01 \\
\hline alfa $_{1}$-globulina & $1.7-5.0$ & $\%$ & 0.01 & $0.02-0.05$ & 1 & $0 . \mathrm{XX}$ & 0.01 \\
\hline alfa $_{2}$-globulina & $6.7-12.5$ & $\%$ & 0.01 & $0.07-0.13$ & 1 & $0 . \mathrm{XX}$ & 0.01 \\
\hline beta-globulina & $8.3-16.3$ & $\%$ & 0.01 & 0.08 .0 .16 & 1 & $0 . \mathrm{XX}$ & 0.01 \\
\hline gama-globulina & $10.7-20.0$ & $\%$ & 0.01 & $0.11-0.20$ & 1 & $0 . \mathrm{XX}$ & 0.01 \\
\hline albúmina & $3.6-5.2$ & $\mathrm{~g} / \mathrm{dL}$ & 10.0 & $36-52$ & $\mathrm{~g} / \mathrm{L}$ & $\mathrm{XX}$ & $1 \mathrm{~g} / \mathrm{L}$ \\
\hline alfa $_{1}$-globulina & $0.1-0.4$ & $\mathrm{~g} / \mathrm{dL}$ & 10.0 & $1-4$ & $g / L$ & $\mathrm{XX}$ & $1 \mathrm{~g} / \mathrm{L}$ \\
\hline alfa $_{2}$-globulina & $0.4-1.0$ & $\mathrm{~g} / \mathrm{dL}$ & 10.0 & $4-10$ & $\mathrm{~g} / \mathrm{L}$ & $\mathrm{XX}$ & $1 \mathrm{~g} / \mathrm{L}$ \\
\hline beta-globulina & $0.5-1.2$ & $\mathrm{~g} / \mathrm{dL}$ & 10.0 & $5-12$ & $\mathrm{~g} / \mathrm{L}$ & $\mathrm{XX}$ & $1 \mathrm{~g} / \mathrm{L}$ \\
\hline gama-globulina & $0.6-1.6$ & $\mathrm{~g} / \mathrm{dL}$ & 10.0 & $6-16$ & $\mathrm{~g} / \mathrm{L}$ & $\mathrm{XX}$ & $1 \mathrm{~g} / \mathrm{L}$ \\
\hline epinefrina $(\mathrm{P})$ & $31-95$ & $\mathrm{pg} / \mathrm{mL}$ & 5.458 & $170-520$ & $\mathrm{pmol} / \mathrm{L}$ & XX0 & $10 \mathrm{pmol} / \mathrm{L}$ \\
\hline epinefrina (o) & $<10$ & $\mu \mathrm{g} / 24 \mathrm{~h}$ & 5.458 & $<55$ & $\mathrm{nmol} / \mathrm{L}$ & $\mathrm{XX}$ & $5 \mathrm{nmol} / \mathrm{d}$ \\
\hline \multicolumn{8}{|l|}{ estradiol (s) } \\
\hline $\begin{array}{l}\text {-hombre mayor de } 18 \text { años } \\
\text { estriol (o) (mujer no embarazada) }\end{array}$ & $15-40$ & $\mathrm{pg} / \mathrm{mL}$ & 3.671 & $55-150$ & pmol & $\mathrm{XXX}$ & $1 \mathrm{pmol} / \mathrm{L}$ \\
\hline comienzo de la menstruación & $4-25$ & $\mu \mathrm{g} / 24 \mathrm{~h}$ & 3.468 & $15-85$ & $\mathrm{nmol} / \mathrm{d}$ & $\mathrm{XXX}$ & $5 \mathrm{nmol} / \mathrm{d}$ \\
\hline pico de ovulación & $28-99$ & $\mu \mathrm{g} / 24 \mathrm{~h}$ & 3.468 & $95-345$ & $\mathrm{nmol} / \mathrm{d}$ & $\mathrm{XXX}$ & $5 \mathrm{nmol} / \mathrm{d}$ \\
\hline pico luterínico & $22-105$ & $\mu \mathrm{g} / 24 \mathrm{~h}$ & 3.468 & $75-365$ & $\mathrm{nmol} / \mathrm{d}$ & $\mathrm{XXX}$ & $5 \mathrm{nmol} / \mathrm{d}$ \\
\hline mujeres menopáusicas & $1.4-19.6$ & $\mu \mathrm{g} / 24 \mathrm{~h}$ & 3.468 & $5-70$ & $\mathrm{nmol} / \mathrm{d}$ & $\mathrm{XXX}$ & $5 \mathrm{nmol} / \mathrm{d}$ \\
\hline hombre & 5-18 & $\mu \mathrm{g} / 24 \mathrm{~h}$ & 3.468 & $15-60$ & $\mathrm{nmol} / \mathrm{d}$ & $\mathrm{XXX}$ & $5 \mathrm{nmol} / \mathrm{d}$ \\
\hline \multicolumn{8}{|l|}{ estrógeno (s) (estradiol) } \\
\hline mujer & $20-300$ & $\mathrm{pg} / \mathrm{mL}$ & 3.671 & $70-1100$ & $\mathrm{pmol} / \mathrm{L}$ & XXX0 & $10 \mathrm{pmol} / \mathrm{L}$ \\
\hline pico de producción & $200-800$ & $\mathrm{pg} / \mathrm{mL}$ & 3.671 & $750-2900$ & $\mathrm{pmol} / \mathrm{L}$ & XXX0 & $10 \mathrm{pmol} / \mathrm{L}$ \\
\hline hombre & $<50$ & $\mathrm{pg} / \mathrm{mL}$ & 3.671 & $<180$ & $\mathrm{pmol} / \mathrm{L}$ & XX0 & $10 \mathrm{pmol} / \mathrm{L}$ \\
\hline \multicolumn{8}{|l|}{ estrógenos, placenta (o) } \\
\hline \multicolumn{8}{|l|}{ receptores estrogénicos $(\mathrm{T})$} \\
\hline negativo & $0-3$ & & 1.00 & $0-3$ & & $\mathrm{XXX}$ & \\
\hline dudoso & $4-10$ & & 1.00 & $4-10$ & & $\mathrm{XXX}$ & \\
\hline positivo & 10 & & 1.00 & 10 & & XXX & \\
\hline \multicolumn{8}{|l|}{ estrona $(\mathrm{P}, \mathrm{s})$} \\
\hline mujer 1-10 días del ciclo & $43-180$ & $\mathrm{pg} / \mathrm{mL}$ & 3.699 & $160-665$ & $\mathrm{pmol} / \mathrm{L}$ & $\mathrm{XXX}$ & $5 \mathrm{pmol} / \mathrm{L}$ \\
\hline mujer $11-12$ días del ciclo & $75-196$ & $\mathrm{pg} / \mathrm{mL}$ & 3.699 & $275-725$ & $\mathrm{pmol} / \mathrm{L}$ & $\mathrm{XXX}$ & $5 \mathrm{pmol} / \mathrm{L}$ \\
\hline mujer 20-39 días del ciclo & $131-201$ & $\mathrm{pg} / \mathrm{mL}$ & 3.699 & 485-745 & $\mathrm{pmol} / \mathrm{L}$ & XXX & $5 \mathrm{pmol} / \mathrm{L}$ \\
\hline hombre & $29-75$ & $\mathrm{pg} / \mathrm{mL}$ & 3.699 & $105-275$ & $\mathrm{pmol} / \mathrm{L}$ & $\mathrm{XXX}$ & $5 \mathrm{pmol} / \mathrm{L}$ \\
\hline
\end{tabular}


APENDICE

Tabla 2. (Continuación) Factores de conversión para los resultados de química clínica.

\begin{tabular}{|c|c|c|c|c|c|c|c|}
\hline Compuesto & $\begin{array}{c}\text { Intervalo de } \\
\text { referencia actual }\end{array}$ & $\begin{array}{l}\text { Unidad } \\
\text { actual }\end{array}$ & $\begin{array}{l}\text { Factores de } \\
\text { conversión }\end{array}$ & $\begin{array}{c}\text { Intervalos } \\
\text { de referencia } \\
\text { SI }\end{array}$ & $\begin{array}{l}\text { Símbolo de } \\
\text { unidad } \\
\text { SI }\end{array}$ & $\begin{array}{c}\text { Dígitos } \\
\text { significativos }\end{array}$ & $\begin{array}{l}\text { Mínimo } \\
\text { incremento } \\
\text { sugerido }\end{array}$ \\
\hline estrona $(0)$ - mujer & $2-25$ & $\mu \mathrm{g} / 24 \mathrm{~h}$ & 3.699 & $5-90$ & $\mathrm{nmol} / \mathrm{d}$ & $\mathrm{XXX}$ & $5 \mathrm{nmol} / \mathrm{d}$ \\
\hline \multicolumn{8}{|l|}{ etanol $(\mathrm{P})$} \\
\hline límite legal (para conducir) & $<80$ & $\mathrm{mg} / \mathrm{dL}$ & 0.2171 & $<17$ & $\mathrm{mmol} / \mathrm{L}$ & $\mathrm{XX}$ & $1 \mathrm{mmol} / \mathrm{L}$ \\
\hline -tóxico & $>100$ & $\mathrm{mg} / \mathrm{dL}$ & 0.2171 & $>22$ & $\mathrm{mmol} / \mathrm{L}$ & $\mathrm{XX}$ & $1 \mathrm{mmol} / \mathrm{L}$ \\
\hline etclovinol (p) - tóxico & $>40$ & $\mathrm{mg} / \mathrm{L}$ & 6.915 & $>280$ & $\mu \mathrm{mol} / \mathrm{L}$ & XX0 & $10 \mu \mathrm{mol} / \mathrm{L}$ \\
\hline \multicolumn{8}{|l|}{ etosuximida (p) } \\
\hline -terapéutico & $40-110$ & $\mathrm{mg} / \mathrm{L}$ & 7.084 & $280-780$ & $\mu \mathrm{mol} / \mathrm{L}$ & XX0 & $10 \mu \mathrm{mol} / \mathrm{L}$ \\
\hline etilenglicol (p) - tóxico & $>30$ & $\mathrm{mg} / \mathrm{L}$ & 0.1611 & $>5$ & $\mathrm{mmol} / \mathrm{L}$ & $\mathrm{XX}$ & $1 \mathrm{mmol} / \mathrm{L}$ \\
\hline grasa (F) (como ácido esteárico) & $2.0-6.0$ & $\mathrm{~g} / 24 \mathrm{~h}$ & 3.515 & $7-21$ & $\mathrm{mmol} / \mathrm{d}$ & $\mathrm{XX}$ & $1 \mathrm{mmol} / \mathrm{d}$ \\
\hline ácidos grasos no esterificados $(\mathrm{P})$ & $8-20$ & $\mathrm{mg} / \mathrm{dL}$ & 10.0 & $80-200$ & $\mathrm{mg} / \mathrm{L}$ & XX0 & $10 \mathrm{mg} / \mathrm{L}$ \\
\hline ferritina (S) & $18-300$ & $\mathrm{ng} / \mathrm{mL}$ & 1.0 & $18-300$ & $\mu \mathrm{g} / \mathrm{L}$ & XX0 & $10 \mu \mathrm{g} / \mathrm{L}$ \\
\hline fibrinógeno (P) & $200-400$ & $\mathrm{mg} / \mathrm{dL}$ & 0.01 & $2.0-4.0$ & $\mathrm{~g} / \mathrm{L}$ & X.X & $0.1 \mathrm{~g} / \mathrm{L}$ \\
\hline fluoruro $(o)$ & $<1.0$ & $\mathrm{mg} / 24 \mathrm{~h}$ & 52.63 & $<50$ & $\mu \mathrm{mol} / \mathrm{d}$ & XX0 & $10 \mu \mathrm{mol} / \mathrm{d}$ \\
\hline \multirow[t]{2}{*}{ folato (s) (como ácido pteroilglutámico } & $2-10$ & $\mathrm{ng} / \mathrm{mL}$ & 2.266 & $4-22$ & $\mathrm{nmol} / \mathrm{L}$ & $\mathrm{XX}$ & $2 \mathrm{nmol} / \mathrm{L}$ \\
\hline & & $\mu \mathrm{g} / \mathrm{dL}$ & 22.66 & & $\mathrm{nmol} / \mathrm{L}$ & & $2 \mathrm{nmol} / \mathrm{L}$ \\
\hline folato (GR) & $140-960$ & $\mathrm{ng} / \mathrm{mL}$ & 2.266 & $550-2200$ & $\mathrm{nmol} / \mathrm{L}$ & XX0 & $10 \mathrm{nmol} / \mathrm{L}$ \\
\hline \multicolumn{8}{|l|}{ hormona folículo estimulante } \\
\hline mujer & $2.0-15.0$ & $\mathrm{mIU} / \mathrm{mL}$ & 1.0 & $2-15$ & $\mathrm{IU} / \mathrm{L}$ & $\mathrm{XX}$ & $1 \mathrm{IU} / \mathrm{d}$ \\
\hline pico de producción & $20-50$ & $\mathrm{mIU} / \mathrm{mL}$ & 1.0 & $20-50$ & $\mathrm{IU} / \mathrm{L}$ & $\mathrm{XX}$ & $1 \mathrm{IU} / \mathrm{d}$ \\
\hline hombre & $1.0-10.0$ & $\mathrm{mIU} / \mathrm{mL}$ & 1.0 & $1-10$ & $\mathrm{IU} / \mathrm{L}$ & $\mathrm{XX}$ & $1 \mathrm{IU} / \mathrm{d}$ \\
\hline \multicolumn{8}{|l|}{ hormona folículo estimulante } \\
\hline FSH $(0)$ & & & & & & & \\
\hline fase folicular & $2-15$ & $\mathrm{IU} / 24 \mathrm{~h}$ & 1.0 & $2-15$ & $\mathrm{IU} / \mathrm{d}$ & $\mathrm{XXX}$ & $1 \mathrm{IU} / \mathrm{d}$ \\
\hline mitad de ciclo & $8-40$ & $\mathrm{IU} / 24 \mathrm{~h}$ & 1.0 & $8-40$ & $\mathrm{IU} / \mathrm{d}$ & $\mathrm{XXX}$ & $1 \mathrm{IU} / \mathrm{d}$ \\
\hline fase luterínica & $2-10$ & $\mathrm{IU} / 24 \mathrm{~h}$ & 1.0 & $2-10$ & $\mathrm{IU} / \mathrm{d}$ & $\mathrm{XXX}$ & $1 \mathrm{IU} / \mathrm{d}$ \\
\hline mujer menopáusica & $35-100$ & $\mathrm{IU} / 24 \mathrm{~h}$ & 1.0 & $35-100$ & $\mathrm{IU} / \mathrm{d}$ & $\mathrm{XXX}$ & $1 \mathrm{IU} / \mathrm{d}$ \\
\hline hombre & $2-15$ & $\mathrm{IU} / 24 \mathrm{~h}$ & 1.0 & $2-15$ & $\mathrm{IU} / \mathrm{d}$ & $\mathrm{XXX}$ & $1 \mathrm{IU} / \mathrm{d}$ \\
\hline fructosa $(\mathrm{P})$ & 10 & $\mathrm{mg} / \mathrm{dL}$ & 0.0551 & 0.6 & $\mathrm{mmol} / \mathrm{L}$ & X.XX & $0.1 \mathrm{nmol} / \mathrm{L}$ \\
\hline galactosa (P) (niños) & 20 & $\mathrm{mg} / \mathrm{dL}$ & 0.0551 & 1.1 & $\mathrm{mmol} / \mathrm{L}$ & X.XX & $0.1 \mathrm{nmol} / \mathrm{L}$ \\
\hline \multicolumn{8}{|l|}{ gases $(\mathrm{S}, \mathrm{a})$} \\
\hline $\mathrm{PO}_{2}$ & $75-105$ & $\mathrm{~mm} \mathrm{Hg}$ & 0.1333 & $10.0-14.0$ & $\mathrm{kPa}$ & XX.X & $0.1 \mathrm{kPa}$ \\
\hline $\mathrm{pCO}_{2}$ & $33-44$ & $\mathrm{~mm} \mathrm{Hg}$ & 0.1333 & 4.4-5.9 & $\mathrm{kPa}$ & X.X & $0.1 \mathrm{kPa}$ \\
\hline \multicolumn{8}{|l|}{ gama-glutamiltransferasa } \\
\hline$(\mathrm{GGT})(\mathrm{s})$ & $0-30$ & $\mathrm{U} / \mathrm{L}$ & 0.01667 & 0.050 & $\mu \mathrm{kat} / \mathrm{L}$ & X.XX & $0.1 \mu \mathrm{kat} / \mathrm{L}$ \\
\hline gastrina (s) & $0-180$ & $\mathrm{pg} / \mathrm{mL}$ & 1 & $0-180$ & $\mathrm{ng} / \mathrm{L}$ & $\mathrm{XX0}$ & $10 \mathrm{ng} / \mathrm{L}$ \\
\hline globulinas (s) (ver inmunoglobulinas) & $\ldots \ldots$ & ...... & ...... & $\ldots \ldots$ & $\ldots \ldots$ & ...... & ...... \\
\hline glucagón (s) & $50-100$ & $\mathrm{pg} / \mathrm{mL}$ & 1 & $50-100$ & $\mathrm{ng} / \mathrm{L}$ & XX0 & $10 \mathrm{ng} / \mathrm{L}$ \\
\hline glucosa $(\mathrm{P})$ - en ayunas & $70-110$ & $\mathrm{mg} / \mathrm{dL}$ & 0.0551 & $3.9-6.1$ & $\mathrm{mmol} / \mathrm{L}$ & XX.X & $0.1 \mathrm{mmol} / \mathrm{L}$ \\
\hline glucosa (LCR) & $50-80$ & $\mathrm{mg} / \mathrm{dL}$ & 0.05551 & $2.8-4.4$ & $\mathrm{mmol} / \mathrm{L}$ & XX.X & $0.1 \mathrm{mmol} / \mathrm{L}$ \\
\hline \multicolumn{8}{|l|}{ glutetimida $(\mathrm{P})$} \\
\hline terapéutico & $<10$ & $\mathrm{mg} / \mathrm{L}$ & 4.603 & $<46$ & $\mu \mathrm{mol} / \mathrm{L}$ & $\mathrm{XX}$ & $1 \mu \mathrm{mol} / \mathrm{L}$ \\
\hline tóxico & $>20$ & $\mathrm{mg} / \mathrm{L}$ & 4.603 & $>92$ & $\mu \mathrm{mol} / \mathrm{L}$ & $\mathrm{XX}$ & $1 \mu \mathrm{mol} / \mathrm{L}$ \\
\hline glicerol, libre (s) & $<1.5$ & $\mathrm{mg} / \mathrm{dL}$ & 0.1086 & $<0.16$ & $\mathrm{mmol} / \mathrm{L}$ & X.XX & $0.01 \mathrm{mmol} / \mathrm{L}$ \\
\hline oro (s) - terapéutico & $300-800$ & $\mu \mathrm{g} / \mathrm{dL}$ & 0.05077 & $15.0-40.0$ & $\mu \mathrm{mol} / \mathrm{L}$ & XX.X & $0.1 \mu \mathrm{mol} / \mathrm{L}$ \\
\hline oro $(\mathrm{o})$ & $<500$ & $\mu \mathrm{g} / 24 \mathrm{~h}$ & 0.005077 & $<2.5$ & $\mu \mathrm{mol} / \mathrm{L}$ & X.X & $0.1 \mu \mathrm{mol} / \mathrm{d}$ \\
\hline \multicolumn{8}{|l|}{ hormona de crecimiento $(\mathrm{P}, \mathrm{s})$} \\
\hline hombre (en ayunas) & $0.0-5.0$ & $\mathrm{ng} / \mathrm{mL}$ & 1.00 & $0.0-5.0$ & $\mu \mathrm{g} / \mathrm{L}$ & XX.X & $0.5 \mu \mathrm{g} / \mathrm{L}$ \\
\hline mujer (en ayunas) & $0.0-10.0$ & $\mathrm{ng} / \mathrm{mL}$ & 1.00 & $0.0-10.0$ & $\mu \mathrm{g} / \mathrm{L}$ & XX.X & $0.5 \mu \mathrm{g} / \mathrm{L}$ \\
\hline haptoglobina (s) & $50-220$ & $\mathrm{mg} / \mathrm{dL}$ & 0.01 & $0.50-2.20$ & $\mathrm{~g} / \mathrm{L}$ & X.XX & $0.01 \mathrm{~g} / \mathrm{L}$ \\
\hline \multicolumn{8}{|l|}{ hemoglobina (S) } \\
\hline hombre & $14.0-18.0$ & $\mathrm{~g} / \mathrm{dL}$ & 10.0 & $140-180$ & $g / L$ & $\mathrm{XXX}$ & $1 \mathrm{~g} / \mathrm{L}$ \\
\hline mujer & $11.5-15.5$ & $\mathrm{~g} / \mathrm{dL}$ & 10.0 & $115-155$ & $\mathrm{gl} / \mathrm{L}$ & $\mathrm{XXX}$ & $1 \mathrm{~g} / \mathrm{L}$ \\
\hline (ver sección de hematología apéndice 1) & & & & & & & \\
\hline ácido homogentísico (o) & 0 & $\mathrm{mg} / 24 \mathrm{~h}$ & 5.947 & 0 & $\mu \mathrm{mol} / \mathrm{d}$ & $\mathrm{XX}$ & $5 \mu \mathrm{mol} / \mathrm{d}$ \\
\hline ácido homovanilico (o) & $<8$ & $\mathrm{mg} / 24 \mathrm{~h}$ & 5.489 & $<45$ & $\mu \mathrm{mol} / \mathrm{d}$ & $\mathrm{XX}$ & $5 \mu \mathrm{mol} / \mathrm{d}$ \\
\hline ácido beta-hidroxibutírico (s) & $<1.0$ & $\mathrm{mg} / \mathrm{dL}$ & 96.05 & $<100$ & $\mu \mathrm{mol} / \mathrm{L}$ & XX0 & $10 \mu \mathrm{mol} / \mathrm{L}$ \\
\hline ácido 5-hidroxindolacético (o) & $2-8$ & $\mathrm{mg} / 24 \mathrm{~h}$ & 5.230 & $10-40$ & $\mu \mathrm{mol} / \mathrm{d}$ & XXX & $5 \mu \mathrm{mol} / \mathrm{d}$ \\
\hline
\end{tabular}


APENDICE

Tabla 2. (Continuación) Factores de conversión para los resultados de química clínica.

\begin{tabular}{|c|c|c|c|c|c|c|c|}
\hline Compuesto & $\begin{array}{c}\text { Intervalo de } \\
\text { referencia actual }\end{array}$ & $\begin{array}{l}\text { Unidad } \\
\text { actual }\end{array}$ & $\begin{array}{l}\text { Factores de } \\
\text { conversión }\end{array}$ & $\begin{array}{c}\text { Intervalos } \\
\text { de referencia } \\
\text { SI }\end{array}$ & $\begin{array}{l}\text { Símbolo de } \\
\text { unidad } \\
\text { SI }\end{array}$ & $\begin{array}{c}\text { Dígitos } \\
\text { significativos }\end{array}$ & $\begin{array}{l}\text { Mínimo } \\
\text { incremento } \\
\text { sugerido }\end{array}$ \\
\hline \multicolumn{8}{|l|}{ 17-alfa-hidroxeprogesterona $(\mathrm{s}, \mathrm{P})$} \\
\hline niños & $0.2-1.4$ & $\mu \mathrm{g} / \mathrm{L}$ & 3.026 & $0.5-4.5$ & $\mathrm{nmol} / \mathrm{L}$ & $\mathrm{XX} . \mathrm{X}$ & $0.5 \mathrm{nmol} / \mathrm{L}$ \\
\hline hombre & $0.5-2.5$ & $\mu \mathrm{g} / \mathrm{L}$ & 3.026 & $1.5-7.5$ & $\mu \mathrm{mol} / \mathrm{L}$ & XX.X & $0.5 \mathrm{nmol} / \mathrm{L}$ \\
\hline mujer & $0.3-4.2$ & $\mu \mathrm{g} / \mathrm{L}$ & 3.026 & $1.0-13.0$ & $\mathrm{nmol} / \mathrm{L}$ & XX.X & $0.5 \mathrm{nmol} / \mathrm{L}$ \\
\hline mujer post-menopáusica & $0.3-1.7$ & $\mu \mathrm{g} / \mathrm{L}$ & 3.026 & $1.0-5.0$ & $\mathrm{nmol} / \mathrm{L}$ & XX.X & $0.5 \mathrm{nmol} / \mathrm{L}$ \\
\hline \multicolumn{8}{|l|}{ hidroxiprolina (o) } \\
\hline-1 semana -1 año & $55-220$ & $\mathrm{mg} / 24 \mathrm{~h} / \mathrm{m}^{2}$ & 7.626 & $420-1680$ & $\mu \mathrm{mol} /\left(\mathrm{d} . \mathrm{m}^{2}\right)$ & $\mathrm{XX0}$ & $10 \mu \mathrm{mol} /\left(\mathrm{d} \cdot \mathrm{m}^{2}\right)$ \\
\hline$-1-13$ años & $25-80$ & $\mathrm{mg} / 24 \mathrm{~h} / \mathrm{m}^{2}$ & 7.626 & $190-610$ & $\mu \mathrm{mol} /\left(\mathrm{d} . \mathrm{m}^{2}\right)$ & $\mathrm{XX0}$ & $10 \mu \mathrm{mol} /\left(\mathrm{d} \cdot \mathrm{m}^{2}\right)$ \\
\hline$-22-65$ años & $6-22$ & $\mathrm{mg} / 24 \mathrm{~h} / \mathrm{m}^{2}$ & 7.626 & $40-170$ & $\mu \mathrm{mol} /\left(\mathrm{d} . \mathrm{m}^{2}\right)$ & XX0 & $10 \mu \mathrm{mol} /\left(\mathrm{d} . \mathrm{m}^{2}\right)$ \\
\hline -mayor de 65 años & $5-17$ & $\mathrm{mg} / 24 \mathrm{~h} / \mathrm{m}^{2}$ & 7.626 & $40-130$ & $\mu \mathrm{mol} /\left(\mathrm{d} \mathrm{m}^{2}\right)$ & $\mathrm{XX0}$ & $10 \mu \mathrm{mol} /\left(\mathrm{d} \cdot \mathrm{m}^{2}\right)$ \\
\hline \multicolumn{8}{|l|}{ inmunoglobulinas (s) } \\
\hline$-\operatorname{IgG}$ & $500-1200$ & $\mathrm{mg} / \mathrm{dL}$ & 0.01 & $5.00-12.00$ & $\mathrm{~g} / \mathrm{L}$ & XX.XX & $0.01 \mathrm{~g} / \mathrm{L}$ \\
\hline$-\operatorname{Ig} \mathrm{A}$ & $50-350$ & $\mathrm{mg} / \mathrm{dL}$ & 0.01 & $0.50-3.50$ & $\mathrm{~g} / \mathrm{L}$ & XX.XX & $0.01 \mathrm{~g} / \mathrm{L}$ \\
\hline$-\operatorname{IgM}$ & $30-230$ & $\mathrm{mg} / \mathrm{dL}$ & 0.01 & $0.30-2.30$ & $\mathrm{~g} / \mathrm{L}$ & XX.XX & $0.01 \mathrm{~g} / \mathrm{L}$ \\
\hline$-\operatorname{IgD}$ & $<6$ & $\mathrm{mg} / \mathrm{dL}$ & 10 & $<60$ & $\mathrm{mg} / \mathrm{L}$ & XX0 & $10 \mathrm{mg} / \mathrm{L}$ \\
\hline -IgE - de 0-3 años & $0.5-10$ & $\mathrm{IU} / \mathrm{mL}$ & 2.4 & $1-24$ & $\mu \mathrm{g} / \mathrm{L}$ & $\mathrm{XX}$ & $1 \mu \mathrm{g} / \mathrm{L}$ \\
\hline de $3-80$ años & $5-100$ & $\mathrm{IU} / \mathrm{mL}$ & 2.4 & $12-240$ & $\mu \mathrm{g} / \mathrm{L}$ & $\mathrm{XX}$ & $1 \mu \mathrm{g} / \mathrm{L}$ \\
\hline imipramina (p)-terapéutica & $50-200$ & $\mathrm{ng} / \mathrm{mL}$ & 3.566 & $189-710$ & $\mathrm{nmol} / \mathrm{L}$ & $\mathrm{XX0}$ & $10 \mathrm{nmol} / \mathrm{L}$ \\
\hline \multirow[t]{3}{*}{ insulina $(\mathrm{P}, \mathrm{s})$} & $5-20$ & $\mu \mathrm{U} / \mathrm{mL}$ & 7.175 & $35-145$ & $\mathrm{pmol} / \mathrm{L}$ & $\mathrm{XXX}$ & $5 \mathrm{pmol} / \mathrm{L}$ \\
\hline & $5-20$ & $\mathrm{mU} / \mathrm{L}$ & 7.175 & $35-145$ & $\mathrm{pmol} / \mathrm{L}$ & $\mathrm{XXX}$ & $5 \mathrm{pmol} / \mathrm{L}$ \\
\hline & $0.20-0.84$ & $\mu \mathrm{g} / \mathrm{mL}$ & 172.2 & $35-145$ & $\mathrm{pmol} / \mathrm{L}$ & $\mathrm{XXX}$ & $5 \mathrm{pmol} / \mathrm{L}$ \\
\hline \multicolumn{8}{|l|}{ hierro (s) } \\
\hline hombre & $80-180$ & $\mu \mathrm{g} / \mathrm{dL}$ & 0.1791 & $14-32$ & $\mu \mathrm{mol} / \mathrm{L}$ & $\mathrm{XX}$ & $1 \mu \mathrm{mol} / \mathrm{L}$ \\
\hline mujer & $60-160$ & $\mu \mathrm{g} / \mathrm{dL}$ & 0.1791 & $11-29$ & $\mu \mathrm{mol} / \mathrm{L}$ & $\mathrm{XX}$ & $1 \mu \mathrm{mol} / \mathrm{L}$ \\
\hline capacidad ligadora de hierro (s) & $250-460$ & $\mu \mathrm{g} / \mathrm{dL}$ & 0.1791 & $45-82$ & $\mu \mathrm{mol} / \mathrm{L}$ & $\mathrm{XX}$ & $1 \mu \mathrm{mol} / \mathrm{L}$ \\
\hline \multicolumn{8}{|l|}{ isoniazida $(\mathrm{p})$} \\
\hline -terapéutica & $<2.0$ & $\mathrm{mg} / \mathrm{L}$ & 7.291 & $<15$ & $\mu \mathrm{mol} / \mathrm{L}$ & $\mathrm{XX}$ & $1 \mu \mathrm{mol} / \mathrm{L}$ \\
\hline -tóxica & $>3.0$ & $\mathrm{mg} / \mathrm{L}$ & 7.291 & $>22$ & $\mu \mathrm{mol} / \mathrm{L}$ & $\mathrm{XX}$ & $1 \mu \mathrm{mol} / \mathrm{L}$ \\
\hline isopropanol (p) & 0 & $\mathrm{mg} / \mathrm{dL}$ & 0.1664 & 0 & $\mathrm{mmol} / \mathrm{L}$ & $\mathrm{XX}$ & $1 \mathrm{mmol} / \mathrm{L}$ \\
\hline \multirow[t]{2}{*}{ lactato (p) (como ácido láctico) } & $0.5-2.0$ & $\mathrm{mEq} / \mathrm{L}$ & 1.00 & $0.5-2.0$ & $\mathrm{mmol} / \mathrm{L}$ & X.X & $0.1 \mathrm{mmol} / \mathrm{L}$ \\
\hline & $5-20$ & $\mathrm{mg} / \mathrm{dL}$ & 0.1110 & $0.5-2.0$ & $\mathrm{mmol} / \mathrm{L}$ & $\mathrm{X} . \mathrm{X}$ & $0.1 \mathrm{mmol} / \mathrm{L}$ \\
\hline lactato dehidrogenasa (s) & $50-150$ & $\mathrm{u} / \mathrm{L}$ & 0.01667 & $0.82-266$ & $\mu \mathrm{kat} / \mathrm{L}$ & X.XX & $0.02 \mu \mathrm{kat} / \mathrm{L}$ \\
\hline \multirow{2}{*}{\multicolumn{8}{|c|}{$\begin{array}{c}\text { lactato dehidrogenasa } \\
\text { isoenzimas (s) }\end{array}$}} \\
\hline & & & & & & & \\
\hline$-\mathrm{LD} 1$ & $15-40$ & $\%$ & 0.01 & $0.15-0.40$ & 1 & $0 . \mathrm{XX}$ & 0.01 \\
\hline -LD2 & $20-45$ & $\%$ & 0.01 & $0.20-0.45$ & 1 & $0 . \mathrm{XX}$ & 0.01 \\
\hline -LD3 & $15-30$ & $\%$ & 0.01 & $0.15-0.30$ & 1 & $0 . \mathrm{XX}$ & 0.01 \\
\hline -LD4 & $5-20$ & $\%$ & 0.01 & $0.05-0.20$ & 1 & $0 . \mathrm{XX}$ & 0.01 \\
\hline -LD5 & $5-20$ & $\%$ & 0.01 & $0.50-0.20$ & 1 & $0 . \mathrm{XX}$ & 0.01 \\
\hline$-\mathrm{LD} 1$ & $10-60$ & $\mathrm{U} / \mathrm{L}$ & 0.0167 & $0.16-1.00$ & $\mu \mathrm{kat} / \mathrm{L}$ & X.XX & $0.02 \mu \mathrm{kat} / \mathrm{L}$ \\
\hline -LD2 & $20-70$ & $\mathrm{U} / \mathrm{L}$ & 0.0167 & $0.32-1.16$ & $\mu \mathrm{kat} / \mathrm{L}$ & X.XX & $0.02 \mu \mathrm{kat} / \mathrm{L}$ \\
\hline -LD3 & $10-45$ & $\mathrm{U} / \mathrm{L}$ & 0.0167 & $0.22-0.76$ & $\mu \mathrm{kat} / \mathrm{L}$ & X.XX & $0.02 \mu \mathrm{kat} / \mathrm{L}$ \\
\hline -LD4 & $5-30$ & $\mathrm{U} / \mathrm{L}$ & 0.0167 & $0.80-0.50$ & $\mu \mathrm{kat} / \mathrm{L}$ & X.XX & $0.02 \mu \mathrm{kat} / \mathrm{L}$ \\
\hline -LD5 & $5-30$ & $\mathrm{U} / \mathrm{L}$ & 0.0167 & $0.02-0.50$ & $\mu \mathrm{kat} / \mathrm{L}$ & X.XX & $0.02 \mu \mathrm{kat} / \mathrm{L}$ \\
\hline plomo (S) - tóxico & $>60$ & $\mu \mathrm{g} / \mathrm{dL}$ & 0.04826 & $>2.90$ & $\mu \mathrm{mol} / \mathrm{L}$ & X.XX & $0.05 \mu \mathrm{mol} / \mathrm{L}$ \\
\hline & & $\mathrm{mg} / \mathrm{dL}$ & 48.26 & & $\mu \mathrm{mol} / \mathrm{L}$ & X.XX & $0.05 \mu \mathrm{mol} / \mathrm{L}$ \\
\hline plomo (o) - tóxico & $>80$ & $\mu \mathrm{g} / 24 \mathrm{~h}$ & 0.004826 & $>0.40$ & $\mu \mathrm{mol} / \mathrm{d}$ & X.XX & $0.05 \mu \mathrm{mol} / \mathrm{d}$ \\
\hline lidocaína (p) (xilocaína) & $1.0-5.0$ & $\mathrm{mg} / \mathrm{L}$ & 4.267 & $4.5-21.5$ & $\mu \mathrm{mol} / \mathrm{L}$ & X.X & $0.5 \mu \mathrm{mol} / \mathrm{L}$ \\
\hline lipasa (s) & $0-160$ & $\mathrm{U} / \mathrm{L}$ & 0.01667 & $0-2.66$ & $\mu \mathrm{kat} / \mathrm{L}$ & X.XX & $0.02 \mu \mathrm{kat} / \mathrm{L}$ \\
\hline lípidos totales (p) & $400-85$ & $\mathrm{mg} / \mathrm{dL}$ & 0.01 & $4.00-8.5$ & $\mathrm{~g} / \mathrm{L}$ & X.X & $0.1 \mathrm{~g} / \mathrm{L}$ \\
\hline \multicolumn{8}{|l|}{ lipoproteínas } \\
\hline de baja densidad (LDL), como colesterol & $50-190$ & $\mathrm{mg} / \mathrm{dL}$ & 0.02586 & $1.30-4.90$ & $\mathrm{mmol} / \mathrm{L}$ & X.XX & $0.05 \mathrm{mmol} / \mathrm{L}$ \\
\hline de alta densidad (DHL), como colesterol & & & & & $\mathrm{mmol} / \mathrm{L}$ & X.XX & $0.05 \mathrm{mmol} / \mathrm{L}$ \\
\hline hombre & $30-70$ & $\mathrm{mg} / \mathrm{dL}$ & 0.02586 & $0.80-1.80$ & $\mathrm{mmol} / \mathrm{L}$ & X.XX & $0.05 \mathrm{mmol} / \mathrm{L}$ \\
\hline mujer & $30-90$ & $\mathrm{mg} / \mathrm{dL}$ & 0.02586 & $0.80-2.35$ & $\mathrm{mmol} / \mathrm{L}$ & X.XX & $0.05 \mathrm{mmol} / \mathrm{L}$ \\
\hline \multirow[t]{3}{*}{ litio iónico (s)-terapéutico } & $0.50-1.50$ & $\mathrm{mEq} / \mathrm{L}$ & 1.00 & $0.50-1.50$ & $\mathrm{mmol} / \mathrm{L}$ & X.XX & $0.05 \mathrm{mmol} / \mathrm{L}$ \\
\hline & & $\mu \mathrm{g} / \mathrm{dL}$ & 0.001441 & & $\mathrm{mmol} / \mathrm{L}$ & X.XX & $0.05 \mathrm{mmol} / \mathrm{L}$ \\
\hline & & $\mathrm{mg} / \mathrm{dL}$ & 1.441 & & $\mathrm{mmo} / \mathrm{L}$ & X.XX & $0.05 \mathrm{mmol} / \mathrm{L}$ \\
\hline \multicolumn{8}{|l|}{ hormona luteinizante (s) } \\
\hline hombre & $3-25$ & $\mathrm{mIU} / \mathrm{mL}$ & 1.00 & $3-25$ & $\mathrm{IU} / \mathrm{L}$ & $\mathrm{XXX}$ & $1 \mathrm{IU} / \mathrm{L}$ \\
\hline mujer & $2-20$ & $\mathrm{mIU} / \mathrm{mL}$ & 1.00 & $2-20$ & $\mathrm{IU} / \mathrm{L}$ & $\mathrm{XXX}$ & $1 \mathrm{U} / \mathrm{L}$ \\
\hline pico de producción & $30-140$ & $\mathrm{mIU} / \mathrm{mL}$ & 1.00 & $30-140$ & $\mathrm{IU} / \mathrm{L}$ & $\mathrm{XXX}$ & IU/L \\
\hline lisozima (s) (muramidasa) & $1-15$ & $\mu \mathrm{g} / \mathrm{mL}$ & 1.00 & $1-15$ & $\mathrm{mg} / \mathrm{L}$ & $\mathrm{XXX}$ & $1 \mathrm{mg} / \mathrm{L}$ \\
\hline
\end{tabular}




\section{APENDICE}

Tabla 2. (Continuación) Factores de conversión para los resultados de química clínica.

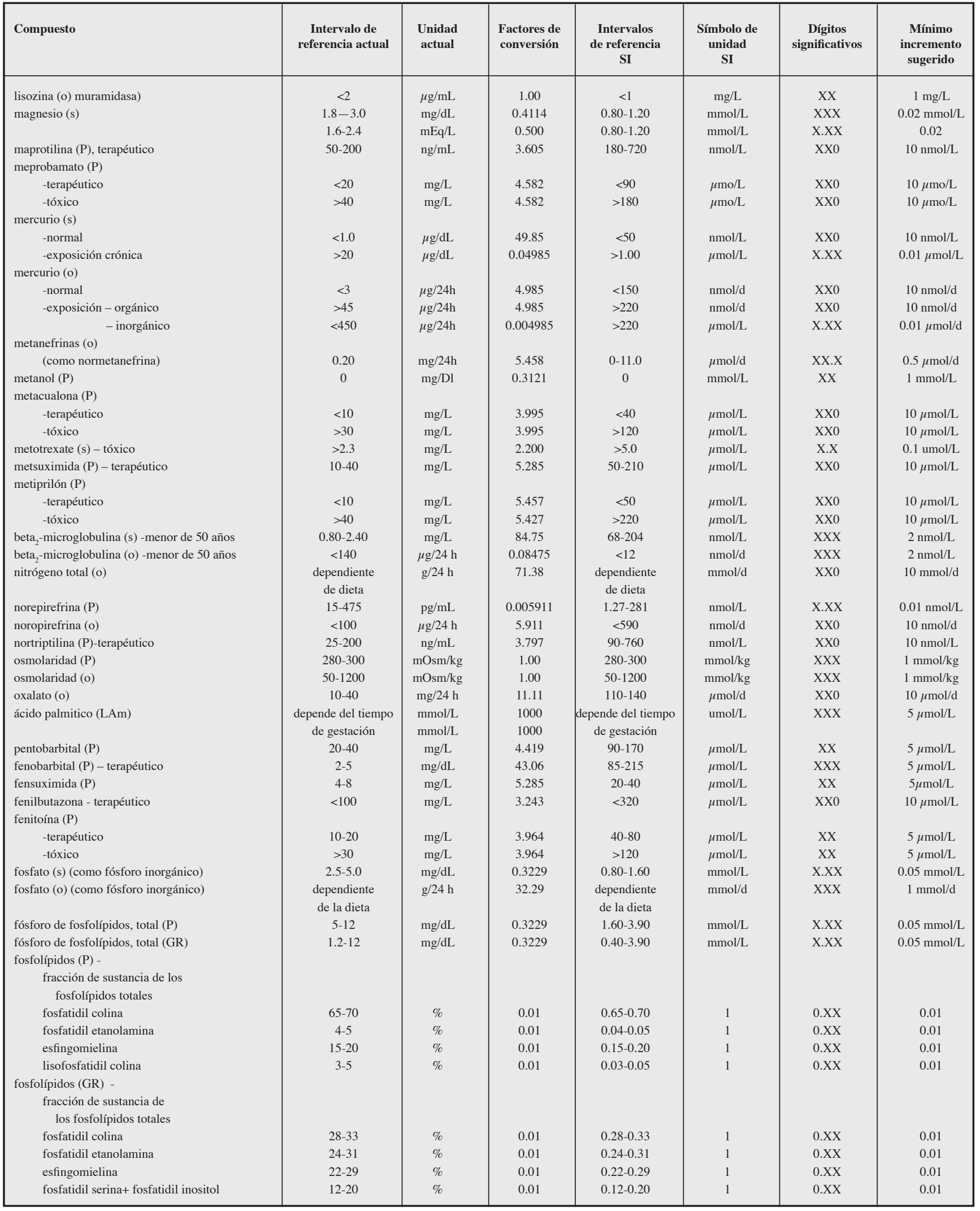




\section{APENDICE}

Tabla 2. (Continuación) Factores de conversión para los resultados de química clínica.

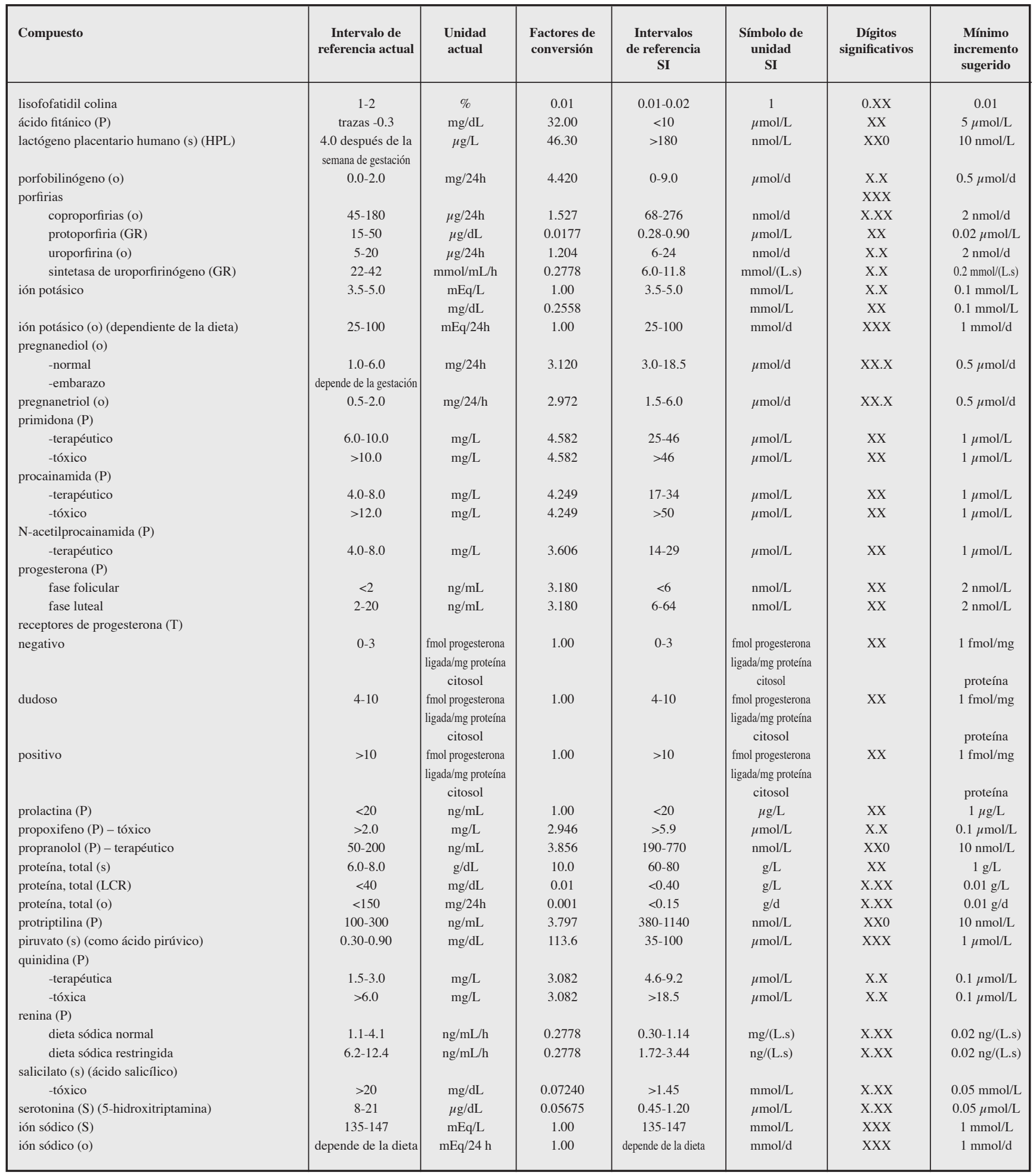


APENDICE

Tabla 2. (Continuación) Factores de conversión para los resultados de química clínica.

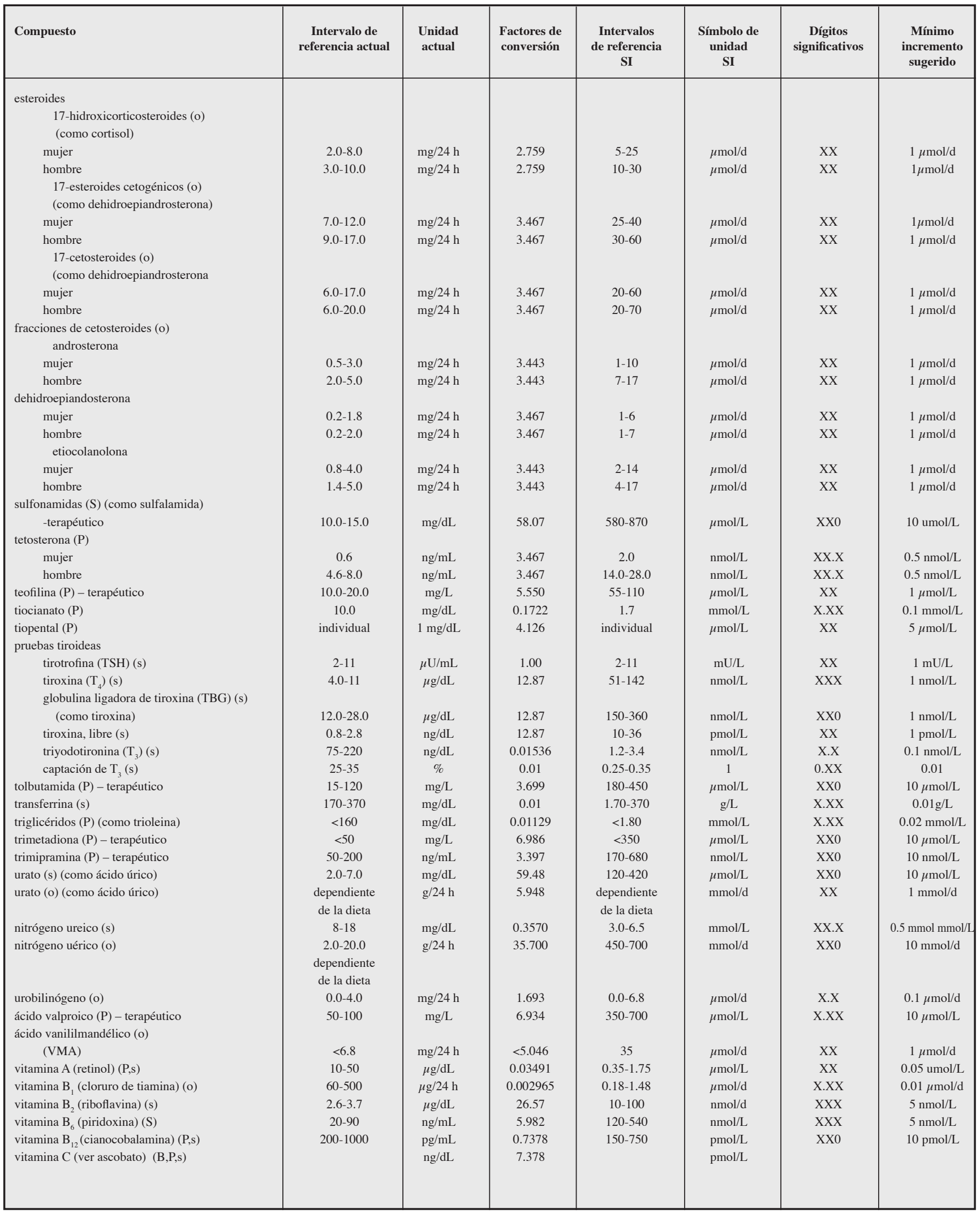




\section{APENDICE}

Tabla 2. (Continuación) Factores de conversión para los resultados de química clínica.

\begin{tabular}{|c|c|c|c|c|c|c|c|}
\hline Compuesto & $\begin{array}{l}\text { Intervalo de } \\
\text { referencia actual }\end{array}$ & $\begin{array}{l}\text { Unidad } \\
\text { actual }\end{array}$ & $\begin{array}{l}\text { Factores de } \\
\text { conversión }\end{array}$ & $\begin{array}{l}\text { Intervalos } \\
\text { de referencia } \\
\text { SI }\end{array}$ & $\begin{array}{l}\text { Símbolo de } \\
\text { unidad } \\
\text { SI }\end{array}$ & $\begin{array}{c}\text { Dígitos } \\
\text { significativos }\end{array}$ & $\begin{array}{c}\text { Mínimo } \\
\text { incremento } \\
\text { sugerido }\end{array}$ \\
\hline vitamina $\mathrm{D}_{3}$ (colecalciferol) $(\mathrm{P})$ & $24-40$ & $\mathrm{ng} / \mathrm{mL}$ & 2.590 & $60-105$ & $\mathrm{nmol} / \mathrm{L}$ & $\mathrm{XXX}$ & $5 \mathrm{nmol} / \mathrm{L}$ \\
\hline 250H-colecalciferol & $18-36$ & $\mathrm{ng} / \mathrm{mL}$ & 2.496 & $45-90$ & $\mathrm{nmol} / \mathrm{L}$ & $\mathrm{XXX}$ & $5 \mathrm{mmol} / \mathrm{L}$ \\
\hline vitamina E (alfa.tocoferol) $(\mathrm{P}, \mathrm{s})$ & $0.78-1.25$ & $\mathrm{mg} / \mathrm{dL}$ & 23.22 & 1829 & $\mu \mathrm{mol} / \mathrm{L}$ & $\mathrm{XX}$ & $1 \mu \mathrm{mol} / \mathrm{L}$ \\
\hline warfarina $(\mathrm{P})$ - terapéutico & $1.0-3.0$ & $\mathrm{mg} / \mathrm{dL}$ & 3.243 & $3.3-9.8$ & $\mu \mathrm{mol} / \mathrm{L}$ & XX.X & $0.1 \mathrm{umol} / \mathrm{L}$ \\
\hline xantina (o) & $5-30$ & $\mathrm{mg} / 24$ & 6.574 & $30-200$ & $\mu \mathrm{mol} / \mathrm{d}$ & XX0 & $10 \mu \mathrm{mol} / \mathrm{d}$ \\
\hline -hipoxantina & & $\mathrm{hmg} / 24 \mathrm{~h}$ & 7.347 & & $\mu \mathrm{mol} / \mathrm{d}$ & XX0 & $10 \mu \mathrm{mol} / \mathrm{d}$ \\
\hline D-xylosa (S) (dosis de $25 \mathrm{~g}$ ) & $30-40$ & $\mathrm{mg} / \mathrm{dL}$ & 0.6661 & $0-2.7$ & $\mathrm{mmol} / \mathrm{L}$ & $\mathrm{X} . \mathrm{X}$ & $0.1 \mathrm{mmol} / \mathrm{L}$ \\
\hline excreción de D-xylosa (o) & (3060 minutos) & & & (30-60 minutos) & & & \\
\hline (dosis de $25 \mathrm{~g}$ ) & 21-31 & @ & 0.01 & 0.21-031 & 1 & $0 . \mathrm{XX}$ & 0.01 \\
\hline zinc (s) & $\begin{array}{c}\text { excreción en } 5 \text { horas } \\
75-120\end{array}$ & $\mu \mathrm{g} / \mathrm{dL}$ & 0.1530 & $\begin{array}{c}\text { excreción en } 5 \text { horas } \\
11.5-18.5\end{array}$ & $\mu \mathrm{mol} / \mathrm{L}$ & XX.X & $0.1 \mu \mathrm{mol} / \mathrm{L}$ \\
\hline zinc (o) & $150-1200$ & $\mu \mathrm{g} / 24 \mathrm{~h}$ & 0.01530 & $2.3-18.3$ & $\mu \mathrm{mol} / \mathrm{d}$ & XX.X & $0.1 \mu \mathrm{mol} / \mathrm{d}$ \\
\hline
\end{tabular}

$\begin{array}{ll}\text { SIGNIFICADO DE LAS ABREVIATURA } \\ \mathrm{S}= & \text { sangre } \\ \mathrm{s}= & \text { suero } \\ \mathrm{p}= & \text { plasma } \\ \mathrm{o}= & \text { orina } \\ \mathrm{LCR}= & \text { Líquido cefalorraquídeo } \\ \mathrm{LAm}= & \text { líquido amniótico } \\ \mathrm{c}= & \text { cabello } \\ \mathrm{T}= & \text { tejido } \\ \mathrm{GR}= & \text { Glóbulos rojos } \\ \mathrm{F}= & \text { eces } \\ \mathrm{a}= & \text { arterial } \\ \mathrm{v}= & \text { venosa } \\ \mathrm{cp}= & \text { capilar } \\ \mathrm{d}= & \text { día }\end{array}$

